\title{
SUPERCRITICAL WATER OXIDATION BENCHSCALE TESTING METALLURGICAL ANALYSIS REPORT
}

\author{
Brad C. Norby \\ February 1993

\section{Idaho National Engineering Laboratory \\ EG\&G Idaho, Inc. Idaho Falls, Idaho}

\section{DISCLAIMER}

This report was prepared as an account of work sponsored by an agency of the United States Government. Neither the United States Government nor any agency thereof, nor any of their employees, makes any warranty, express or implied, or assumes any legal liability or responsibility for the accuracy, completeness, or usefulness of any information, apparatus, product, or process disclosed, or represents that its use would not infringe privately owned rights. Reference herein to any specific commercial product, process, or service by trade name, trademark, manufacturer, or otherwise does not necessarily constitute or imply its endorsement, recommendation, or favoring by the United States Government or any agency thereof. The views and opinions of authors expressed herein do not necessarily state or reflect those of the United States Government or any agency thereof.

\section{Prepared for the \\ U.S. Department of Energy \\ Office of Technology Development \\ Under DOE Idaho Field Office \\ Contract DE-AC07-76ID01570}




\section{DISCLAMMIRR}

Portions of this document may be illegible in electronic image products. Images are produced from the best available original document. 


\section{ABSTRACT}

This report describes metallurgical evaluation of witness wires from a series of tests using supercritical water oxidation ( $\mathrm{SCWO}$ ) to process cutting oil containing a simulated radionuclide. The goal of the tests was to evaluate the technology's ability to process a highly chlorinated waste representative of many mixed waste streams generated in the DOE complex. The testing was conducted with a bench-scale SCWO system developed by the Modell Development Corporation. Significant test objectives included process optimization for adequate destruction efficiency, tracking the radionuclide simulant and certain metals in the effluent streams, and assessment of reactor material degradation resulting from processing a highly chlorinated waste. The metallurgical evaluation described herein includes results of metallographic analysis and Scanning Electron Microscopy analysis of witness wires exposed to the SCWO environment for one test series. 


\section{CONTENTS}

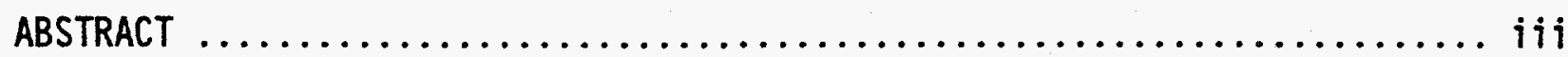

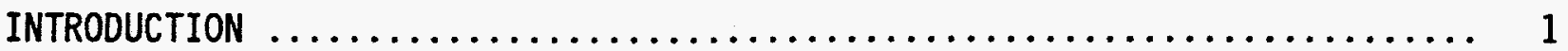

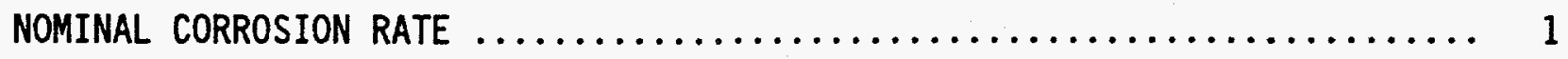

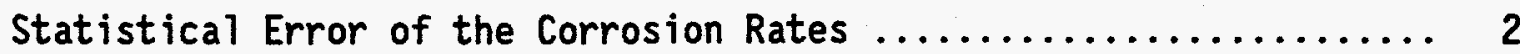
Corrosion Rate Calculations ................................... ${ }_{2}$

Effect of Deposit on Corrosion Rate .......................... 3

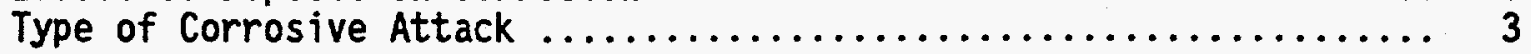

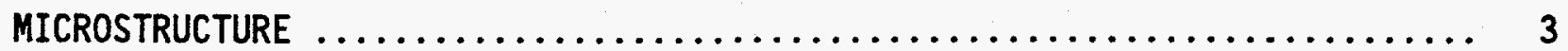

SCANNING ELECTRON MICROSCOPY $\ldots \ldots \ldots \ldots \ldots \ldots \ldots \ldots \ldots \ldots \ldots \ldots \ldots \ldots \ldots \ldots \ldots, 4$

CONCLUSIONS $\ldots \ldots \ldots \ldots \ldots \ldots \ldots \ldots \ldots \ldots \ldots \ldots \ldots \ldots \ldots \ldots \ldots \ldots \ldots \ldots, 4$

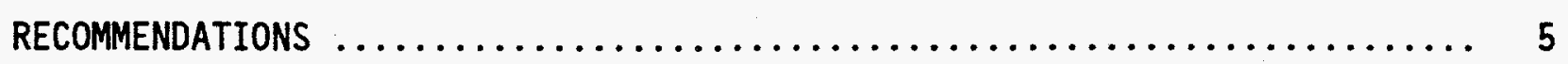

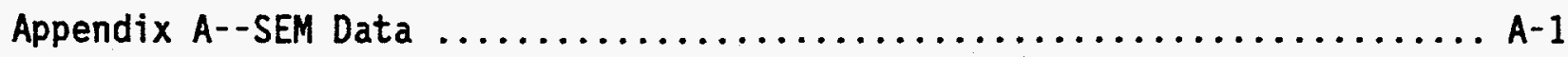




\section{FIGURES}

1. SCWO Reactor Corrosion Inconel 62540 hour test ................. 14

2. SCWO Reactor Corrosion Inconel 62520 hour test ................. 15

3. Original diameter of sensitized wire between 19.5 and $20.0 \mathrm{ft} \ldots \ldots \ldots 16$

4. Wire diameter after 20 hours exposure in the reactor

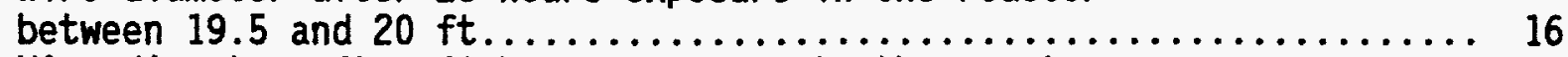

5. Wire diameter after 40 hours exposure to the reactor

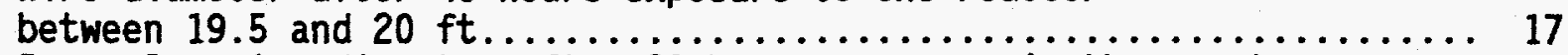

6. Irregular wire diameter after 20 hours exposure in the reactor

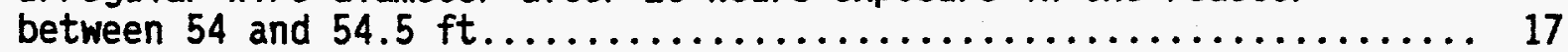

7. Irregular wire diameter after exposure in the reactor

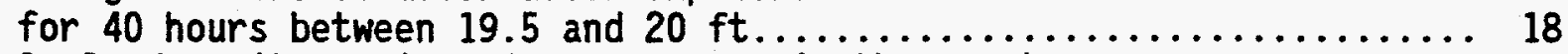

8. Scale deposit on wire after exposure in the reactor

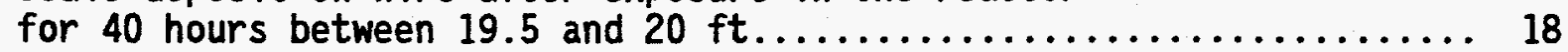

9. Intergranular attack of wire after exposure in the reactor

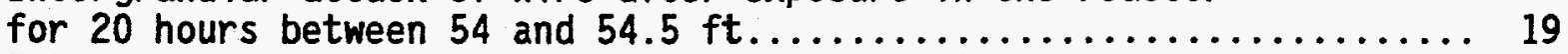

10. Intergranular attack of wires after exposure in the reactor for 40 hours between 18.5 and $19 \mathrm{ft} \ldots \ldots \ldots \ldots \ldots \ldots \ldots \ldots \ldots \ldots \ldots . \ldots \ldots$

11. General attack of wires exposed in the reactor for 40 hours

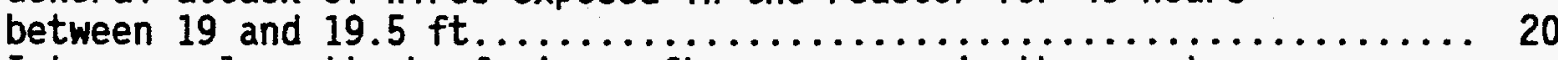

12. Intergranular attack of wires after exposure in the reactor

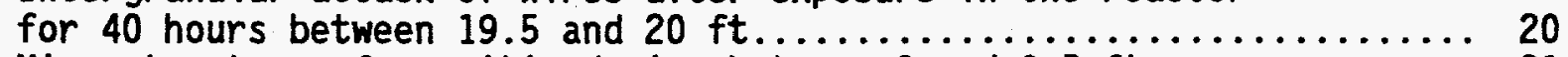

13. Microstructure of sensitized wire between 9 and $9.5 \mathrm{ft} \ldots \ldots \ldots \ldots \ldots, 21$

14. Edges of sensitized wires located between 19.5 to $20 \mathrm{ft}$. showing the thin oxide layer and possible precipitates $\ldots \ldots \ldots \ldots \ldots 21$

15. Sensitized wires taken from between 9 and $9.5 \mathrm{ft}$. showing the difference in grain sizes $\ldots \ldots \ldots \ldots \ldots \ldots \ldots \ldots \ldots, 22$

16. SEM photograph showing scale and intergranular attack $\ldots \ldots \ldots \ldots \ldots .23$

17. SEM photograph of the wire in Figure 16 at higher magnification ..... 23

18. SEM photograph showing large deposit on one side of wire and

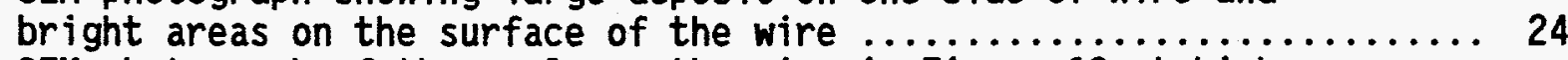

19. SEM photograph of the scale on the wire in Figure 18 at higher

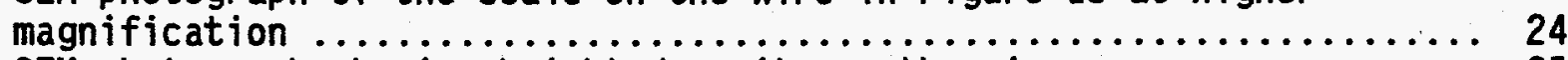

20. SEM photograph showing bright deposits on the wires $\ldots \ldots \ldots \ldots \ldots \ldots 25$

21. SEM photograph of the bright scale on the wire in Figure 20

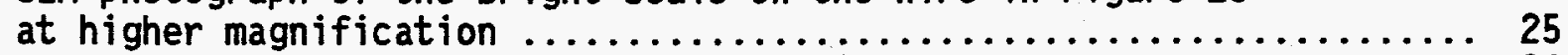

22. SEM photograph showing dark scale deposit .................... 26

23. SEM photograph of deposit on the wire in Figure 22 at higher

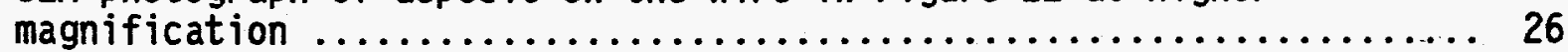

24. SEM photograph showing continuous deposit around the wire $\ldots \ldots \ldots \ldots .27$

25. SEM photograph of deposit on the wire in Figure 24 at higher

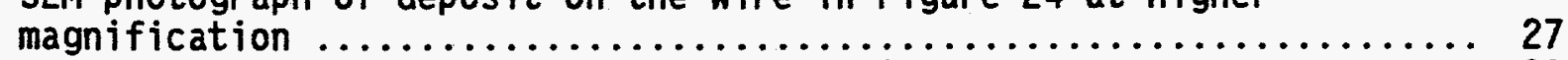

26. SEM photograph showing i ittle scale deposit ..................... 28

27. SEM photograph of deposit on the wire in Figure 26 at higher

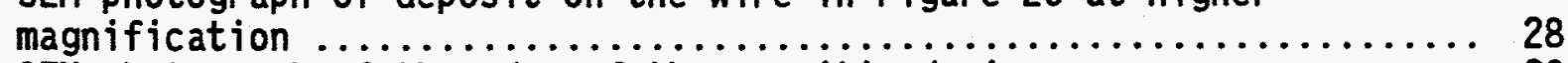

28. SEM photograph of the edge of the sensitized wire $\ldots \ldots \ldots \ldots \ldots \ldots, 29$

29. SEM photograph showing large oxide area $\ldots \ldots \ldots \ldots \ldots \ldots \ldots \ldots \ldots .29$

30. SEM photograph of deposit on the wire in Figure 29 at higher

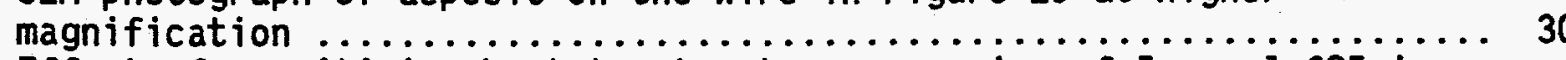

31. Effect of sensitizing heat treatment on corrosion of Incone 1625 in

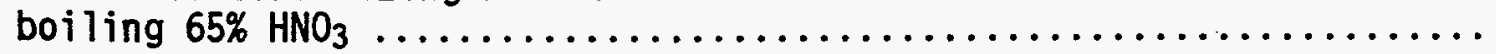




\section{TABLES}

1. Supercritical Water Oxidation Thickness Measurements Incone 1625 Wire Exposed for 20 hours $\ldots \ldots \ldots \ldots \ldots \ldots \ldots \ldots . \ldots . \ldots .6$

2. Supercritical Water Oxidation Thickness Measurements Incone 1625 Wire Exposed for 40 hours $\ldots \ldots \ldots \ldots \ldots \ldots \ldots \ldots \ldots . \ldots \ldots$ 


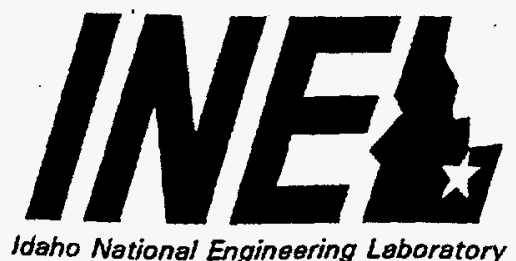

$\mathrm{BCN}-01-93$

January 28, 1993

Carolyn Shapiro, Engineering Specialist

EG\&G Idaho Inc.

P.0. Box 1625

Idaho Falls, ID 83415-3416

\section{Introduction}

Metallographic analys is and Scanning Electron Microscopy of Inconel 625 weld wires have been completed. These wires were exposed to the corrosive environment of the Supercritical Water Oxidation (SCWO) reactor.

Three weld wires were examined. All three wires were placed in the reactor and sensitized. The sensitizing heat treatment was $800^{\circ} \mathrm{C}$ for 24 hours. This sensitization heat treatment was inaccurately considered a passivation treatment. After the sensitizing heat treatment, one wire was removed from the reactor and the other two were exposed to the reactor environment for 20 and 40 hours respectively.

After exposure the wires were removed from the reactor. Between 68 and 80 samples were cut from each wire at various locations along the wire length and then prepared for metallographic examination.

The objective of the study was to get an estimate of the corrosion rate at various locations in the reactor, to determine the type of corrosive attack, to determine the effect of the sensitization heat treatment, and to determine the composition and effect of deposits that formed on the wires.

Nominal Corrosion Rate

\section{Method for Determining the Corrosion Rate}

Gravimetric (weight loss) methods were not used to determine the corrosion rate because it was not possible to place small corrosion coupons throughout the reactor. As an alternative, the diameters of the weld wires placed in the reactor were measured using the metallograph. The three weld wires ran the entire length of the reactor and as such allowed for an estimate of the corrosion rate in all areas of the reactor. 
BCN-01-93

Carolyn Shapiro

January 28, 1993

Page 2

Determining corrosion rates using thickness measurements are not as accurate as gravimetric methods. However, it is possible to get an estimate of corrosion rate if the rates are high.

\section{Statistical Error of the Corrosion Rates}

The nominal thickness of the original weld wires before heat treating was 0.0625 inch. Measurements of the diameter of the sensitized wire (the wire that was not exposed to the corrosive fluids) at 68 locations along the length of the wire, gave a mean value of 0.0612 inches. The standard deviation for the diameter was 0.0003 inches. Therefore, the $95 \%$ confidence interval for the true mean diameter was from 0.0606 inches to 0.0618 inches.

The units used for the calculated corrosion rates were mpy, or 0.001 "/year. The calculated confidence interval is equivalent to $+/-70 \mathrm{mpy}$. Therefore, the reported corrosion rates could vary by as much as $70 \mathrm{mpy}$. Reported corrosion rates less than 70 mpy could easily be attributed to measurement errors and variations in the wire diameter, not actual corrosion loss.

Corrosion Rate Calculations

The estimated corrosion rates along with the estimated metal loss for each wire are listed in Tables 1 and 2. The corrosion rates are also plotted in Figures 1 and 2 . In several areas of the reactor the corrosion rates were high enough that the metal loss could be attributed to corrosion and not just variations in the weld wire diameter.

The corrosion rates were extremely high from about the 12 foot area of the reactor to the 26 foot area. Figures 3 through 5 show the progressive attack of the wires during operation of the reactor. The areas of active corrosion were between about the 10 and 37 foot area of the reactor and at the end of the reactor. At the 54 foot area the wire exposed for 20 hours experienced rates in excess of $2000 \mathrm{mpy}$ (Figure 6). A similar event occurred at the 53.5 foot area and the 54 foot area on the wire that was exposed for 40 hours.

Materials with general corrosion rates less than 2 mpy are generally considered to have good corrosion resistance and considered compatible with the environment. As a common rule, corrosion rates in excess of 20 mpy are typically considered unacceptably high for typical engineering structures fabricated from corrosion resistant alloys.

The corrosion rates from the 55 to 60 foot area of the reactor could not be calculated because the wires were not available. 
$\mathrm{BCN}-01-93$

Carolyn Shapiro

January 28, 1993

Page 3

\section{Effect of Deposit on Corrosion Rate}

In some areas the corroded wires were irregularly shaped indicating localized corrosion (Figures 6 and 7). The corrosion rates seemed to be effected by deposits that formed on the wires. These deposits seemed to form a protective coating and hence slow down the corrosion. Apparently, corrosion would occur in a specific area then layers of scale would begin to form on the wires. The corrosion would slow (or stop) until the scale spalled off. This would explain why, in general, the corrosion rates for the wire exposed to the reactor for 20 hours had higher corrosion rates than the wire exposed for 40 hours. The appearance of the scale was similar to sedimentary rocks with distinct layers (Figure 8 ).

Type of Corrosive Attack

Most of the areas of the wires experienced general corrosion. However, some areas of the wires experienced intergranular attack (Figure 9). Intergranular attack is a form of localized corrosion where corrosion occurs more rapidly at the grain boundaries.

The degree of intergranular attack varied within short distances. Wire segments adjacent to each other experienced different degrees of intergranular attack (compare Figures 10, 11, and 12). Since the degree of sensitization would be relatively uniform, the variance in intergranular attack would indicate different chemical environments within short distances along the reactor.

\section{Microstructure}

The microstructure of the weld wires showed equiaxed grains with twinning, typical for Inconel 625 (Figure 13). This microstructure is typical of the solution annealed condition (that is, heated to about $2100^{\circ} \mathrm{F}$ and water quenched). There were no obvious indications of secondary precipitates caused by the sensitization heat treatment. However, there were some secondary phase precipitates (or maybe just oxide scale) concentrated near the edges of the weld wires (Figure 14). There was no indication of grain distortion indicative of cold working.

The grain size varied along the length of the weld wire. This difference in size occurred within inches. Figure 15 shows the difference in grain size between two samples that were taken one half inch apart from each other.

The microstructure of the weld wire was similar to any plate, pipe, or tubing that would have been hot worked, solution annealed, and sensitized. The nominal chemical composition of Inconel 625 weld wire is identical to the 
$\mathrm{BCN}-01-93$

Carolyn Shapiro

January 28, 1993

Page 4

nominal chemical composition of Inconel 625 plate. Similar composition and similar microstructure mean that the weld wires most likely behaved the same as the reactor walls.

\section{Scanning Electron Microscopy}

The chemical composition of the deposits were analyzed using Energy Dispersive $X$-ray Spectrometry (EDS) of the Scanning Electron Microscope (SEM). The composition was determined for the deposits at the 6.5 to 7.0 foot location, the 19.5 to 20 foot location, and the 53.5 to 54 foot location. Twenty six different spectra were run.

In general, the deposits on the wires included oxides of the elements that compose Inconel 625 (see spectra 1-4 and Figures 16-17). In addition to these oxides, cerium, zinc, lead, chloride, and sulfur were found in the deposits. Sulfur was only found in spectra 5.

Of the three wire locations analyzed, the cerium and lead were concentrated at the 6.5 to 7 foot level (see spectra 8, 12, and 13 and Figures 18-21). Small amounts of cerium and lead were also detected at the 19.5 to 20 foot location and the 53.5 to 54 foot location (see spectra $6,7,17$, and 18 and Figures 2225). Zinc was only detected at the 53.5 to 54 foot location (see spectra 17 , 18,20 , and 21 and Figures 24-27). Chloride was detected in all three analyzed areas (Spectra 7-11 and 16-18). High chloride nodules were found imbedded in the deposit at the 6.5 to 7 foot area (Spectra 10, Figure 19).

Depending on the valence, small amounts of cerium in the waste would accelerate the corrosion. Cerium $(+4)$ is a strong oxidizer. The oxidation potential of $\mathrm{Ce}^{+3}$ to $\mathrm{Ce}^{+4}$ is -1.61 volts. The presence of cerium could also enhance intergranular attack.

The composition of the oxide layer on the sensitized wire consisted of high concentrations of chromium oxide (Spectra 22-25, Figures 28-30). There was one area on the sensitized wire that was composed of nickel oxide (Spectrum 26).

The high concentrations of aluminum were most likely due to the polishing compound used for metallographic sample preparation.

\section{Conclusions}

1. Both intergranular and general attack occurs throughout the reactor. Intergranular attack seems to be inconsistent in its location. Two segments of wire $1 / 2$ inch away from each other could experience general and 
$\mathrm{BCN}-01-93$

Carolyn Shapiro

January 29, 1993

Page 5

intergranular attack. The intergranular attack was most likely enhanced by the sensitization heat treatment and the presence of cerium in the waste.

2. The deposit that forms on the reactor provides partial protection from the corrosive atmosphere. However, this deposit is not tightly adhering and can not be counted on to reduce corrosion.

3. Incone 625 experiences heavy corrosion. In general the area of extensive corrosion is between the 12 foot and 26 feet area of the reactor and at the end of the reactor in the cool down section.

4. The sensitizing heat treatment that the reactor experienced was detrimental to the corrosion resistance of Inconel 625 (Figure 31).

5. The deposits on the wires consisted of oxides of the elements that compose Inconel 625. The deposits also included cerium, lead, zinc, chloride, and sulfur.

\section{Recommendations}

1. Inconel 625 is not a suitable alloy for the tested environment. A different alloy should be used. Depending on the reactor environment Hastelloy $\mathrm{C}-22$ or Hastelloy $\mathrm{G}-30$ could provide better corrosion resistance. Hastelloy $\mathrm{C}-22$ has performed well in halide solutions both in reducing environments and those with low levels of oxidants. Hastelloy G-30 has performed well in oxidizing solutions with halide contaminants.

2. If Inconel 625 is used for continued pilot plant tests, do not sensitize the material prior to running the tests.

3. Replace the reactor prior to any further testing.

4. If possible, it would be advantageous to examine the reactor parts in the heavily corroded areas.

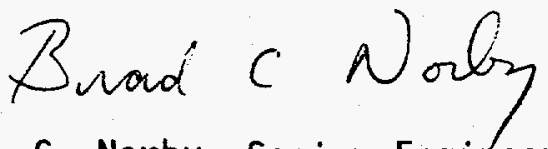

B. C. Norby, Senior Engineer Materials Development and Technology

Attachment

$/ d j$ 


\begin{tabular}{|c|c|c|c|c|c|}
\hline \multicolumn{6}{|c|}{$\begin{array}{c}\text { Supercritical Water Oxidation Thickness Measurements } \\
\text { Inconel } 625 \text { Wire Exposed for } 20 \text { Hours }\end{array}$} \\
\hline $\begin{array}{l}\text { Wire Location } \\
\text { in Reactor }\end{array}$ & $\begin{array}{c}\text { Wire } \\
\text { Sample } \\
\text { Number }\end{array}$ & $\begin{array}{l}\text { Sensitized } \\
\text { Wire } \\
\text { Thickness } \\
\text { (inches) }\end{array}$ & $\begin{array}{l}\text { Wire } \\
\text { Thickness } \\
\text { after } \\
\text { Exposure } \\
\text { (inches) }\end{array}$ & $\begin{array}{c}\text { Estimated } \\
\text { Metal Loss } \\
(0.001 ")\end{array}$ & $\begin{array}{c}\text { Estimated } \\
\text { Corrosion } \\
\text { Rate } \\
\text { (mpy) }\end{array}$ \\
\hline \multirow[t]{4}{*}{ 5'6"-6'0" } & 1 & 0.062 & 0.0612 & 0.02 & 7 \\
\hline & 2 & 0.061 & 0.0616 & 0.00 & 0 \\
\hline & 3 & 0.061 & 0.0616 & 0.00 & 0 \\
\hline & 4 & 0.061 & 0.0616 & 0.00 & 0 \\
\hline \multirow[t]{4}{*}{ 6'6"-7’0" } & 1 & 0.061 & 0.062 & 0.00 & 0 \\
\hline & 2 & 0.061 & 0.0612 & 0.02 & 7 \\
\hline & 3 & 0.0612 & 0.0622 & 0.00 & 0 \\
\hline & 4 & 0.0612 & 0.0616 & 0.00 & 0 \\
\hline \multirow[t]{4}{*}{$7^{\prime} 6^{\prime \prime}-8^{\prime} 0^{\prime \prime}$} & 1 & 0.0614 & 0.061 & 0.12 & 51 \\
\hline & 2 & 0.0616 & 0.061 & 0.12 & 51 \\
\hline & 3 & 0.0616 & 0.0608 & 0.22 & 95 \\
\hline & 4 & 0.0614 & 0.061 & 0.12 & 51 \\
\hline \multirow[t]{4}{*}{ 9'0"-9'6" } & 1 & 0.0612 & 0.0612 & 0.02 & 7 \\
\hline & 2 & 0.061 & 0.0612 & 0.02 & 7 \\
\hline & 3 & 0.0612 & 0.0612 & 0.02 & 7 \\
\hline & 4 & 0.0612 & 0.061 & 0.12 & 51 \\
\hline \multirow[t]{4}{*}{ 9'6"-10’0" } & 1 & 0.0612 & & & \\
\hline & 2 & 0.0612 & & & \\
\hline & 3 & 0.0612 & & & \\
\hline & 4 & 0.0614 & & & \\
\hline \multirow[t]{2}{*}{$12^{\prime} 0^{\prime \prime}-12^{\prime} 8^{\prime \prime}$} & 1 & 0.0614 & 0.057 & 2.12 & 927 \\
\hline & 2 & 0.0612 & 0.053 & 4.12 & 1803 \\
\hline
\end{tabular}




\begin{tabular}{|c|c|c|c|c|c|}
\hline \multicolumn{6}{|c|}{$\begin{array}{c}\text { Table } 1 \\
\text { Supercritical Water Oxidation Thickness Measurements } \\
\text { Inconel } 625 \text { Wire Exposed for } 20 \text { Hours }\end{array}$} \\
\hline \multirow[t]{3}{*}{$\begin{array}{l}\text { Wire Location } \\
\text { in Reactor }\end{array}$} & $\begin{array}{c}\text { Wire } \\
\text { Sample } \\
\text { Number }\end{array}$ & $\begin{array}{l}\text { Sensitized } \\
\text { Wire } \\
\text { Thickness } \\
\text { (inches) }\end{array}$ & $\begin{array}{l}\text { Wire } \\
\text { Thickness } \\
\text { after } \\
\text { Exposure } \\
\text { (inches) }\end{array}$ & $\begin{array}{l}\text { Estimated } \\
\text { Metal Loss } \\
\left(0.001^{\prime \prime}\right)\end{array}$ & $\begin{array}{c}\text { Estimated } \\
\text { Corrosion } \\
\text { Rate } \\
\text { (mpy) }\end{array}$ \\
\hline & 3 & 0.0614 & 0.052 & 4.62 & 2022 \\
\hline & 4 & 0.061 & 0.058 & 1.62 & 708 \\
\hline \multirow[t]{4}{*}{$16^{\prime} 0^{\prime \prime}-16^{\prime} 6^{\prime \prime}$} & 1 & 0.0608 & 0.057 & 2.12 & 927 \\
\hline & 2 & 0.0612 & 0.057 & 2.12 & 927 \\
\hline & 3 & 0.061 & 0.056 & 2.62 & 1146 \\
\hline & 4 & 0.061 & 0.056 & 2.62 & 1146 \\
\hline \multirow[t]{4}{*}{$18^{\prime} 0^{\prime \prime}-18^{\prime} 6^{\prime \prime}$} & 1 & 0.0612 & 0.0596 & 0.82 & 357 \\
\hline & 2 & 0.0614 & 0.0592 & 1.02 & 445 \\
\hline & 3 & 0.061 & 0.059 & 1.12 & 489 \\
\hline & 4 & 0.0608 & 0.06 & 0.62 & 270 \\
\hline \multirow[t]{4}{*}{$18^{\prime} 6^{\prime \prime}-19^{\prime} 0^{\prime \prime}$} & 1 & 0.0608 & 0.058 & 1.62 & 708 \\
\hline & 2 & 0.061 & 0.055 & 3.12 & 1365 \\
\hline & 3 & 0.0612 & 0.056 & 2.62 & 1146 \\
\hline & 4 & 0.061 & 0.053 & 4.12 & 1803 \\
\hline \multirow[t]{4}{*}{ 19'0"-19'6" } & 1 & 0.0612 & 0.054 & 3.62 & 1584 \\
\hline & 2 & 0.0604 & 0.0546 & 3.32 & 1452 \\
\hline & 3 & 0.0612 & 0.054 & 3.62 & 1584 \\
\hline & 4 & 0.061 & 0.055 & 3.12 & 1365 \\
\hline \multirow[t]{4}{*}{$19^{\prime} 6^{\prime \prime}-20^{\prime} 0^{\prime \prime}$} & 1 & 0.0612 & 0.0554 & 2.92 & 1277 \\
\hline & 2 & 0.0608 & 0.057 & 2.12 & 927 \\
\hline & 3 & 0.0614 & 0.0512 & 5.02 & 2197 \\
\hline & 4 & 0.0608 & 0.052 & 4.62 & 2022 \\
\hline $25^{\prime} 0^{\prime \prime}-25^{\prime} 6^{\prime \prime}$ & 1 & 0.0614 & 0.055 & 3.12 & 1365 \\
\hline
\end{tabular}


Table 1

Supercritical Water Oxidation Thickness Measurements Inconel 625 Wire Exposed for 20 Hours

\begin{tabular}{|c|c|c|c|c|c|}
\hline $\begin{array}{l}\text { Wire Location } \\
\text { in Reactor }\end{array}$ & $\begin{array}{c}\text { Wire } \\
\text { Sample } \\
\text { Number }\end{array}$ & $\begin{array}{l}\text { Sensitized } \\
\text { Wire } \\
\text { Thickness } \\
\text { (inches) }\end{array}$ & $\begin{array}{l}\text { Wire } \\
\text { Thickness } \\
\text { after } \\
\text { Exposure } \\
\text { (inches) }\end{array}$ & $\begin{array}{c}\text { Estimated } \\
\text { Metal Loss } \\
\left(0.001^{\prime \prime}\right)\end{array}$ & $\begin{array}{c}\text { Estimated } \\
\text { Corrosion } \\
\text { Rate } \\
\text { (mpy) }\end{array}$ \\
\hline & 2 & 0.0616 & 0.053 & 4.12 & 1803 \\
\hline & 3 & 0.0614 & 0.0534 & 3.92 & 1715 \\
\hline & 4 & 0.0616 & 0.054 & 3.62 & 1584 \\
\hline \multirow[t]{4}{*}{$25^{\prime} 6^{\prime \prime}-26^{\prime} 0^{\prime \prime}$} & 1 & 0.0612 & 0.053 & 4.12 & 1803 \\
\hline & 2 & 0.0614 & 0.059 & 1.12 & 489 \\
\hline & 3 & 0.0618 & 0.06 & 0.62 & 270 \\
\hline & 4 & 0.062 & 0.059 & 1.12 & 489 \\
\hline \multirow[t]{4}{*}{$30^{\prime} 0^{\prime \prime}-30^{\prime} 6^{\prime \prime}$} & 1 & 0.0608 & 0.06 & 0.62 & 270 \\
\hline & 2 & 0.0608 & 0.06 & 0.62 & 270 \\
\hline & 3 & 0.0618 & 0.0596 & 0.82 & 357 \\
\hline & 4 & 0.061 & 0.0598 & 0.72 & 314 \\
\hline \multirow[t]{4}{*}{$37^{\prime} 0^{\prime \prime}-37^{\prime} 6^{\prime \prime}$} & 1 & 0.061 & 0.06 & 0.62 & 270 \\
\hline & 2 & 0.0612 & 0.062 & 0.00 & 0 \\
\hline & 3 & 0.0612 & 0.061 & 0.12 & 51 \\
\hline & 4 & 0.061 & 0.0604 & 0.42 & 182 \\
\hline \multirow[t]{4}{*}{$38^{\prime} 0^{\prime \prime}-38^{\prime} 6^{\prime \prime}$} & 1 & & 0.061 & 0.12 & 51 \\
\hline & 2 & & 0.061 & 0.12 & 51 \\
\hline & 3 & & 0.061 & 0.12 & 51 \\
\hline & 4 & & 0.061 & 0.12 & 51 \\
\hline \multirow[t]{4}{*}{$47^{\prime} 6^{\prime \prime}-48^{\prime} 0^{\prime \prime}$} & 1 & & 0.061 & 0.12 & 51 \\
\hline & 2 & & 0.061 & 0.12 & 51 \\
\hline & 3 & & 0.061 & 0.12 & 51 \\
\hline & 4 & & 0.061 & 0.12 & 51 \\
\hline
\end{tabular}


Table 1

Supercritical Water Oxidation Thickness Measurements Inconel 625 Wire Exposed for 20 Hours

\begin{tabular}{|c|c|c|c|c|c|}
\hline $\begin{array}{l}\text { Wire Location } \\
\text { in Reactor }\end{array}$ & $\begin{array}{c}\text { Wire } \\
\text { Sample } \\
\text { Number }\end{array}$ & $\begin{array}{l}\text { Sensitized } \\
\text { Wire } \\
\text { Thickness } \\
\text { (inches) }\end{array}$ & $\begin{array}{l}\text { Wire } \\
\text { Thickness } \\
\text { after } \\
\text { Exposure } \\
\text { (inches) }\end{array}$ & $\begin{array}{c}\text { Estimated } \\
\text { Metal Loss } \\
\left(0.001^{\prime \prime}\right)\end{array}$ & $\begin{array}{c}\text { Estimated } \\
\text { Corrosion } \\
\text { Rate } \\
\text { (mpy) }\end{array}$ \\
\hline \multirow[t]{4}{*}{$52^{\prime} 0^{\prime \prime}-52^{\prime} 6^{\prime \prime}$} & 1 & & 0.063 & 0 & 0 \\
\hline & 2 & & 0.061 & 0.12 & 51 \\
\hline & 3 & & 0.061 & 0.12 & 51 \\
\hline & 4 & & 0.0605 & 0.73 & 160 \\
\hline \multirow[t]{4}{*}{53 '6"-54'0" } & 1 & 0.0614 & 0.0604 & 0.42 & 182 \\
\hline & 2 & 0.0616 & 0.0602 & 0.52 & 226 \\
\hline & 3 & 0.0614 & 0.061 & 0.12 & 51 \\
\hline & 4 & 0.0614 & 0.0602 & 0.52 & 226 \\
\hline \multirow[t]{4}{*}{$54^{\prime} 0^{\prime \prime}-54^{\prime} 6^{\prime \prime}$} & 1 & 0.0616 & 0.049 & 6.12 & 2679 \\
\hline & 2 & 0.0616 & 0.049 & 6.12 & 2679 \\
\hline & 3 & 0.0616 & 0.051 & 5.12 & 2241 \\
\hline & 4 & 0.0616 & 0.057 & 2.12 & 927 \\
\hline \multicolumn{2}{|c|}{ Average } & 0.06123 & & & \\
\hline \multicolumn{2}{|c|}{ Standard Deviation } & 0.00030 & & & \\
\hline \multicolumn{2}{|c|}{ Variance } & $9.25 \times 10^{-8}$ & & & \\
\hline
\end{tabular}




\begin{tabular}{|c|c|c|c|c|c|}
\hline \multicolumn{6}{|c|}{$\begin{array}{l}\text { Table } 2 \\
\text { ater Oxidation Thickness Measurements } \\
525 \text { Wire Exposed for } 40 \text { Hours }\end{array}$} \\
\hline $\begin{array}{l}\text { Wire Location } \\
\text { in Reactor }\end{array}$ & $\begin{array}{c}\text { Wire } \\
\text { Sample } \\
\text { Number }\end{array}$ & $\begin{array}{l}\text { Sensitized } \\
\text { Wire } \\
\text { Thickness } \\
\text { (inches) }\end{array}$ & $\begin{array}{l}\text { Wire } \\
\text { Thickness } \\
\text { after } \\
\text { Exposure } \\
\text { (inches) }\end{array}$ & $\begin{array}{c}\text { Estimated } \\
\text { Metal Loss } \\
\left(0.001^{\prime \prime}\right)\end{array}$ & $\begin{array}{c}\text { Estimated } \\
\text { Corrosion } \\
\text { Rate } \\
\text { (mpy) }\end{array}$ \\
\hline \multirow[t]{4}{*}{$5^{\prime} 6^{\prime \prime}-6^{\prime} 0^{\prime \prime}$} & 1 & 0.062 & 0.061 & 0.12 & 25 \\
\hline & 2 & 0.061 & 0.0612 & 0.02 & 4 \\
\hline & 3 & 0.061 & 0.0614 & 0.00 & 0 \\
\hline & 4 & 0.061 & 0.0612 & 0.02 & 4 \\
\hline \multirow[t]{4}{*}{ 6'6"-7’0" } & 1 & 0.061 & 0.061 & 0.12 & 25 \\
\hline & 2 & 0.061 & 0.061 & 0.12 & 25 \\
\hline & 3 & 0.0612 & 0.061 & 0.12 & 25 \\
\hline & 4 & 0.0612 & 0.0608 & 0.22 & 47 \\
\hline \multirow[t]{4}{*}{$7^{\prime} 6^{\prime \prime}-8^{\prime} 0^{\prime \prime}$} & 1 & 0.0614 & 0.0614 & 0.00 & 0 \\
\hline & 2 & 0.0616 & 0.0616 & 0.00 & 0 \\
\hline & 3 & 0.0616 & 0.0612 & 0.02 & 4 \\
\hline & 4 & 0.0614 & 0.061 & 0.12 & 25 \\
\hline \multirow[t]{4}{*}{ 9'0"-9'6" } & 1 & 0.0612 & 0.0612 & 0.02 & 4 \\
\hline & 2 & 0.061 & 0.0612 & 0.02 & 4 \\
\hline & 3 & 0.0612 & 0.0612 & 0.02 & 4 \\
\hline & 4 & 0.0612 & 0.061 & 0.12 & 25 \\
\hline \multirow[t]{4}{*}{ 9'6"-10’0" } & 1 & 0.0612 & 0.06 & 0.62 & 135 \\
\hline & 2 & 0.0612 & 0.0602 & 0.52 & 113 \\
\hline & 3 & 0.0612 & 0.0604 & 0.42 & 91 \\
\hline & 4 & 0.0614 & 0.0602 & 0.52 & 113 \\
\hline \multirow[t]{2}{*}{$12^{\prime} 0^{\prime \prime}-12^{\prime} 8^{\prime \prime}$} & 1 & 0.0614 & 0.05 & 5.62 & 1230 \\
\hline & 2 & 0.0612 & 0.048 & 6.62 & 1449 \\
\hline
\end{tabular}


Table 2

Supercritical Water Oxidation Thickness Measurements Inconel 625 Wire Exposed for 40 Hours

\begin{tabular}{|c|c|c|c|c|c|}
\hline $\begin{array}{l}\text { Wire Location } \\
\text { in Reactor }\end{array}$ & $\begin{array}{c}\text { Wire } \\
\text { Sample } \\
\text { Number }\end{array}$ & $\begin{array}{l}\text { Sensitized } \\
\text { Wire } \\
\text { Thickness } \\
\text { (inches) }\end{array}$ & $\begin{array}{c}\text { Wire } \\
\text { Thickness } \\
\text { after } \\
\text { Exposure } \\
\text { (inches) }\end{array}$ & $\begin{array}{c}\text { Estimated } \\
\text { Metal Loss } \\
(0.001 ")\end{array}$ & $\begin{array}{c}\text { Estimated } \\
\text { Corrosion } \\
\text { Rate } \\
\text { (mpy) }\end{array}$ \\
\hline & 3 & 0.0614 & 0.054 & 3.62 & 792 \\
\hline & 4 & 0.061 & 0.054 & 3.62 & 792 \\
\hline \multirow[t]{4}{*}{$16^{\prime} 0^{\prime \prime}-16^{\prime} 6^{\prime \prime}$} & 1 & 0.0608 & 0.0544 & 3.42 & 748 \\
\hline & 2 & 0.0612 & 0.055 & 3.12 & 682 \\
\hline & 3 & 0.061 & 0.053 & 4.12 & 901 \\
\hline & 4 & 0.061 & 0.053 & 4.12 & 901 \\
\hline \multirow[t]{4}{*}{$18^{\prime} 0^{\prime \prime}-18^{\prime} 6^{\prime \prime}$} & 1 & 0.0612 & 0.054 & 3.62 & 792 \\
\hline & 2 & 0.0614 & 0.052 & 4.62 & 1011 \\
\hline & 3 & 0.061 & 0.046 & 7.62 & 1668 \\
\hline & 4 & 0.0608 & 0.05 & 5.62 & 1230 \\
\hline \multirow[t]{4}{*}{$18^{\prime} 6^{\prime \prime}-19^{\prime} 0^{\prime \prime}$} & 1 & 0.0608 & 0.052 & 4.62 & 1011 \\
\hline & 2 & 0.061 & 0.053 & 4.12 & 901 \\
\hline & 3 & 0.0612 & 0.05 & 5.62 & 1230 \\
\hline & 4 & 0.061 & 0.048 & 6.62 & 1449 \\
\hline \multirow[t]{4}{*}{ 19’0"-19'6" } & 1 & 0.0612 & 0.052 & 4.62 & 1011 \\
\hline & 2 & 0.0604 & 0.045 & 8.12 & 1777 \\
\hline & 3 & 0.0612 & 0.048 & 6.62 & 1449 \\
\hline & 4 & 0.061 & 0.047 & 7.12 & 1558 \\
\hline \multirow[t]{4}{*}{ 19'6"-20’0" } & 1 & 0.0612 & 0.04 & 10.62 & 2325 \\
\hline & 2 & 0.0608 & 0.05 & 5.62 & 1230 \\
\hline & 3 & 0.0614 & 0.051 & 5.12 & 1120 \\
\hline & 4 & 0.0608 & 0.051 & 5.12 & 1120 \\
\hline $25^{\prime} 0^{\prime \prime}-25^{\prime} 6^{\prime \prime}$ & 1 & 0.0614 & 0.054 & 3.62 & 792 \\
\hline
\end{tabular}




\begin{tabular}{|c|c|c|c|c|c|}
\hline & $\begin{array}{r}\text { Supercritic } \\
\text { Ince }\end{array}$ & $\begin{array}{l}\text { Ta } \\
\text { Water Oxidat } \\
\text { el } 625 \text { Wire }\end{array}$ & $\begin{array}{l}2 \\
\text { Thickness } \\
\text { osed for } 40\end{array}$ & $\begin{array}{l}\text { easurements } \\
\text { ours }\end{array}$ & \\
\hline $\begin{array}{l}\text { Wire Location } \\
\text { in Reactor }\end{array}$ & $\begin{array}{c}\text { Wire } \\
\text { Sample } \\
\text { Number }\end{array}$ & $\begin{array}{l}\text { Sensitized } \\
\text { Wire } \\
\text { Thickness } \\
\text { (inches) }\end{array}$ & $\begin{array}{c}\text { Wire } \\
\text { Thickness } \\
\text { after } \\
\text { Exposure } \\
\text { (inches) }\end{array}$ & $\begin{array}{l}\text { Estimated } \\
\text { Metal Loss } \\
\left(0.001^{\prime \prime}\right)\end{array}$ & $\begin{array}{c}\text { Estimated } \\
\text { Corrosion } \\
\text { Rate } \\
\text { (mpy) }\end{array}$ \\
\hline & 2 & 0.0616 & 0.054 & 3.62 & 792 \\
\hline & 3 & 0.0614 & 0.058 & 1.62 & 354 \\
\hline & 4 & 0.0616 & 0.051 & 5.12 & 1120 \\
\hline $25^{\prime} 6^{\prime \prime}-26^{\prime} 0^{\prime \prime}$ & 1 & 0.0612 & 0.06 & 0.62 & 135 \\
\hline & 2 & 0.0614 & 0.044 & 8.62 & 1887 \\
\hline & 3 & 0.0618 & 0.048 & 6.62 & 1449 \\
\hline & 4 & 0.062 & 0.054 & 3.62 & 792 \\
\hline $30^{\prime} 0^{\prime \prime}-30^{\prime} 6^{\prime \prime}$ & 1 & 0.0608 & 0.0596 & 0.82 & 179 \\
\hline & 2 & 0.0608 & 0.0594 & 0.92 & 201 \\
\hline & 3 & 0.0618 & 0.06 & 0.62 & 135 \\
\hline & 4 & 0.061 & 0.0594 & 0.92 & 201 \\
\hline $37^{\prime} 0^{\prime \prime}-37^{\prime} 6^{\prime \prime}$ & 1 & 0.061 & 0.6 & 0.00 & 0 \\
\hline & 2 & 0.0612 & 0.6 & 0.00 & 0 \\
\hline & 3 & 0.0612 & 0.0604 & 0.42 & 91 \\
\hline & 4 & 0.061 & 0.06 & 0.62 & 135 \\
\hline $38^{\prime} 0^{\prime \prime}-38^{\prime} 6^{\prime \prime}$ & 1 & & 0.0605 & 0.37 & 80 \\
\hline & 2 & & 0.06 & 0.62 & 135 \\
\hline & 3 & & 0.0602 & 0.52 & 113 \\
\hline & 4 & & 0.0602 & 0.52 & 113 \\
\hline $47^{\prime} 6^{\prime \prime}-48^{\prime} 0^{\prime \prime}$ & 1 & & 0.0605 & 0.37 & 80 \\
\hline & 2 & & 0.0605 & 0.37 & 80 \\
\hline & 3 & & 0.0606 & 0.32 & 69 \\
\hline & 4 & & 0.0605 & 0.37 & 80 \\
\hline
\end{tabular}


Table 2

Supercritical Water Oxidation Thickness Measurements Inconel 625 Wire Exposed for 40 Hours

\begin{tabular}{|c|c|c|c|c|c|}
\hline $\begin{array}{l}\text { Wire Location } \\
\text { in Reactor }\end{array}$ & $\begin{array}{c}\text { Wire } \\
\text { Sample } \\
\text { Number }\end{array}$ & $\begin{array}{l}\text { Sensitized } \\
\text { Wire } \\
\text { Thickness } \\
\text { (inches) }\end{array}$ & $\begin{array}{l}\text { Wire } \\
\text { Thickness } \\
\text { after } \\
\text { Exposure } \\
\text { (inches) }\end{array}$ & $\begin{array}{c}\text { Estimated } \\
\text { Metal Loss } \\
(0.001 ")\end{array}$ & $\begin{array}{c}\text { Estimated } \\
\text { Corrosion } \\
\text { Rate } \\
\text { (mpy) }\end{array}$ \\
\hline \multirow[t]{4}{*}{$52^{\prime} 0^{\prime \prime}-52^{\prime} 6^{\prime \prime}$} & 1 & & 0.061 & 0.12 & 25 \\
\hline & 2 & & 0.061 & 0.12 & 25 \\
\hline & 3 & & 0.060 & 0.62 & 135 \\
\hline & 4 & & 0.0605 & 0.37 & 80 \\
\hline \multirow[t]{5}{*}{$53^{\prime} 6^{\prime \prime}-54^{\prime} 0^{\prime \prime}$} & 1 & 0.0614 & 0.0602 & 0.52 & 113 \\
\hline & 2 & 0.0616 & 0.06 & 0.62 & 135 \\
\hline & 3 & 0.0614 & 0.057 & 2.12 & 463 \\
\hline & 4 & 0.0614 & 0.058 & 1.62 & 354 \\
\hline & 5 & & 0.039 & 11.12 & 2427 \\
\hline \multirow[t]{5}{*}{$54^{\prime} 0^{\prime \prime}-54^{\prime} 6^{\prime \prime}$} & 1 & 0.0616 & 0.061 & 0.12 & 25 \\
\hline & 2 & 0.0616 & 0.061 & 0.12 & 25 \\
\hline & 3 & 0.0616 & 0.061 & 0.12 & 25 \\
\hline & 4 & 0.0616 & 0.061 & 0.12 & 25 \\
\hline & 5 & & 0.055 & 3.12 & 681 \\
\hline \multicolumn{2}{|c|}{ Average } & 0.06123 & & & \\
\hline \multicolumn{2}{|c|}{ Standard Deviation } & 0.00030 & & & \\
\hline \multicolumn{2}{|c|}{ Variance } & $9.25 \times 10^{-8}$ & & & \\
\hline
\end{tabular}




\section{SCWO Reactor Corrosion}

\section{Inconel 62540 hour test}

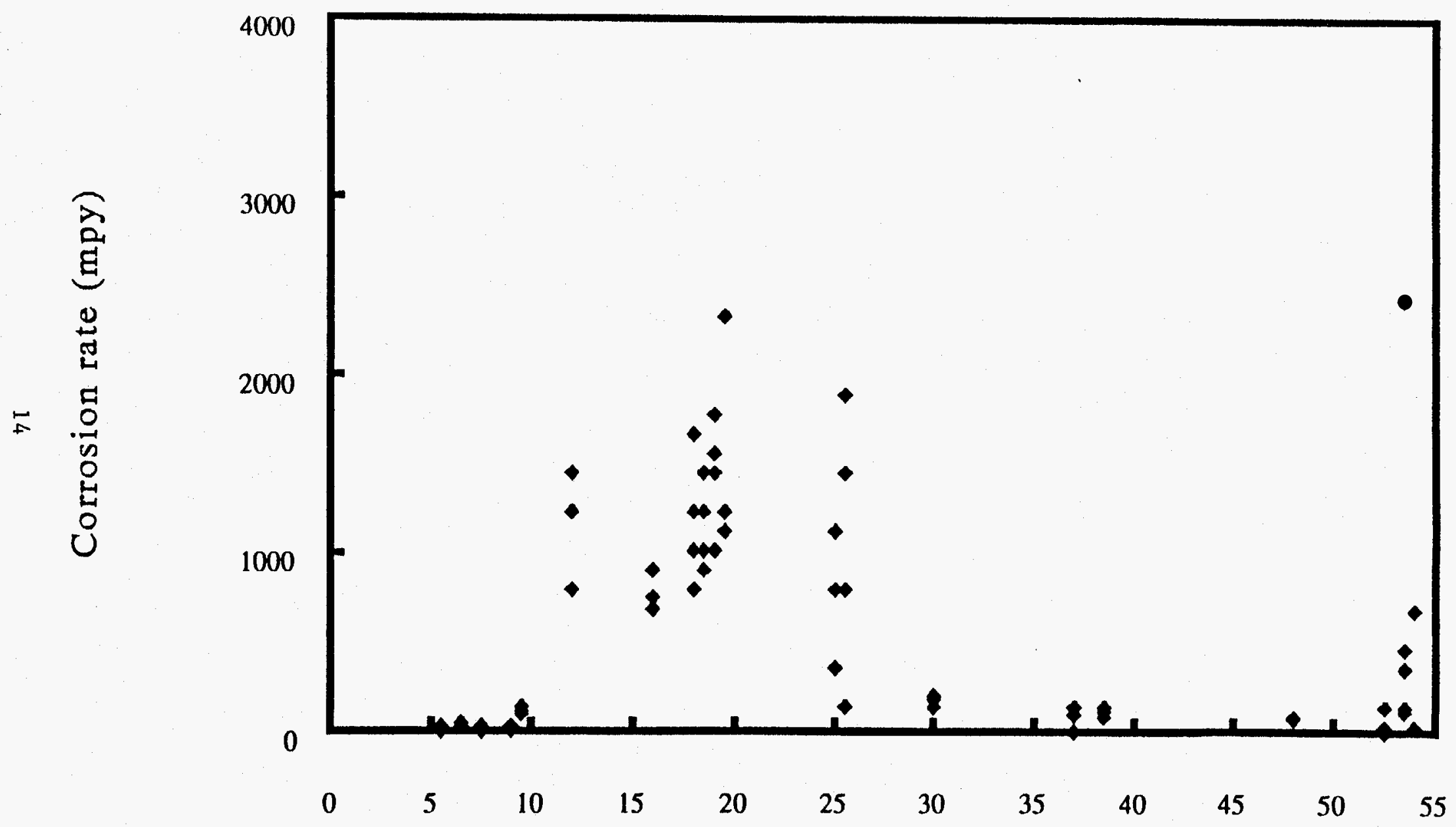

Reactor Location (feet)

Figure 1 


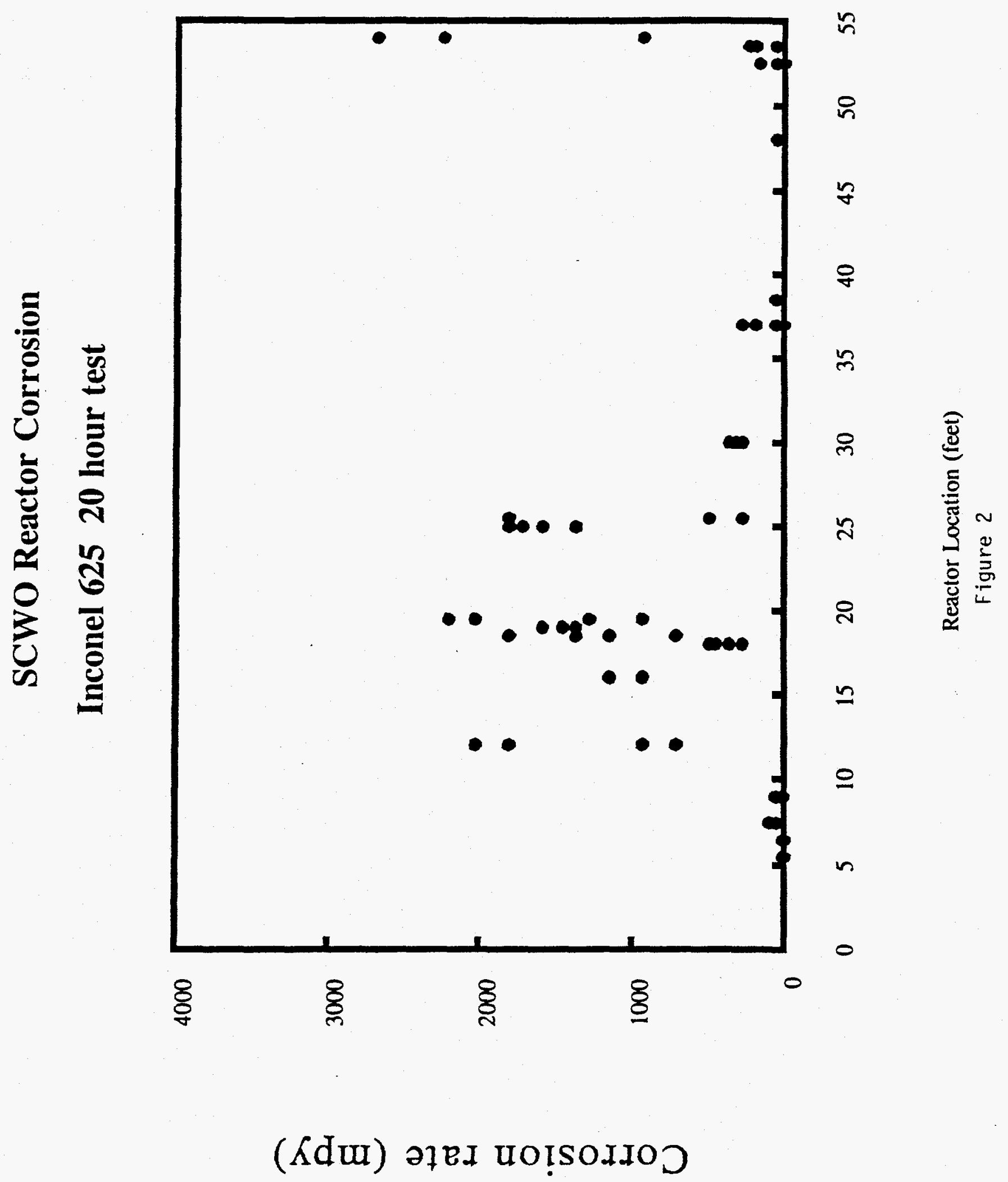




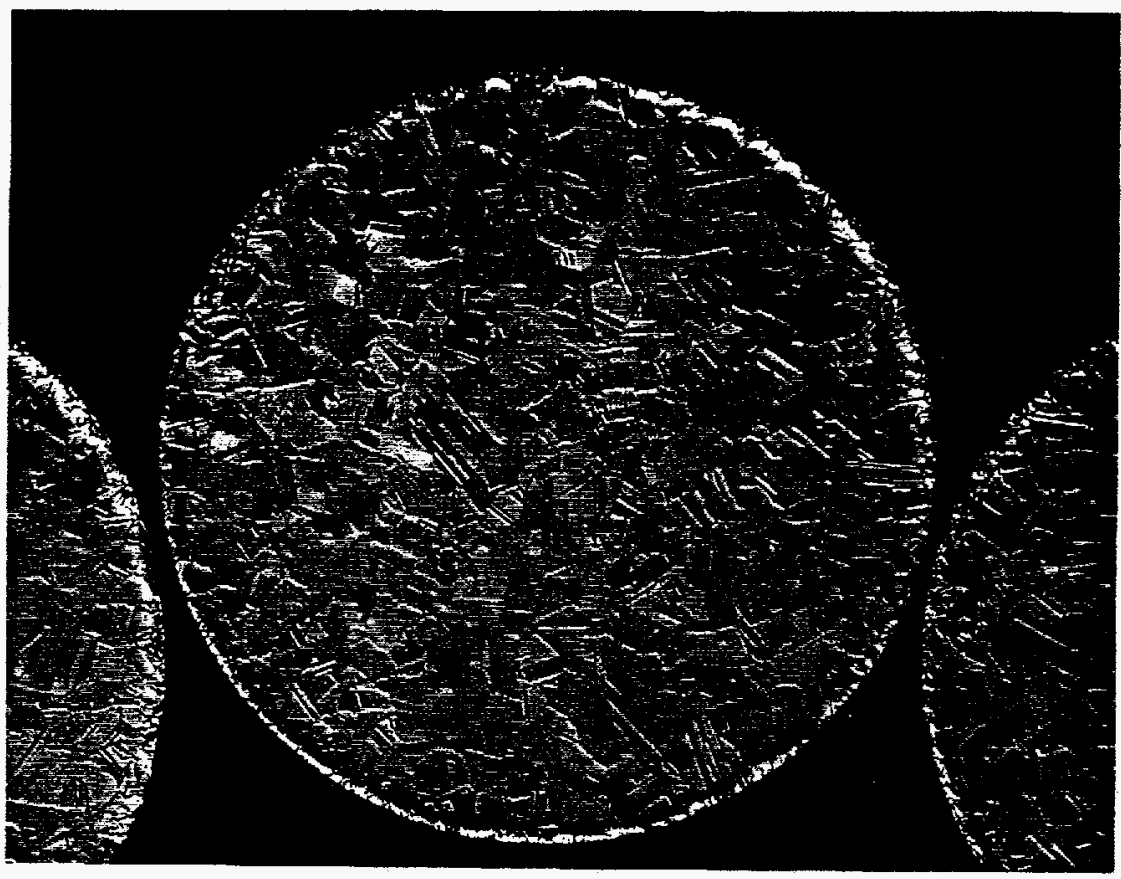

Figure 3

Original diameter of sensitized wire between 19.5 and $20.0 \mathrm{ft}$. (50X as polished)

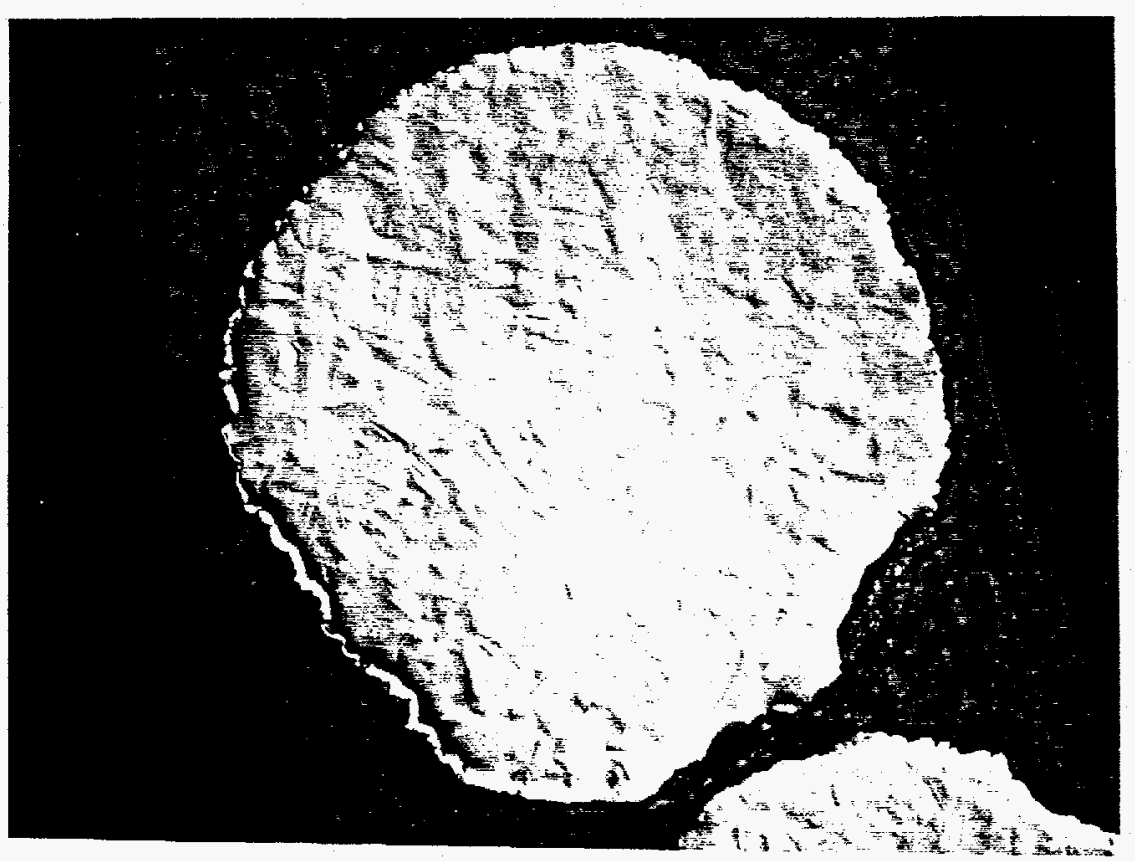

Figure 4

Wire diameter after 20 hours exposure in the reactor between 19.5 and $20 \mathrm{ft}$. (50X as polished) 


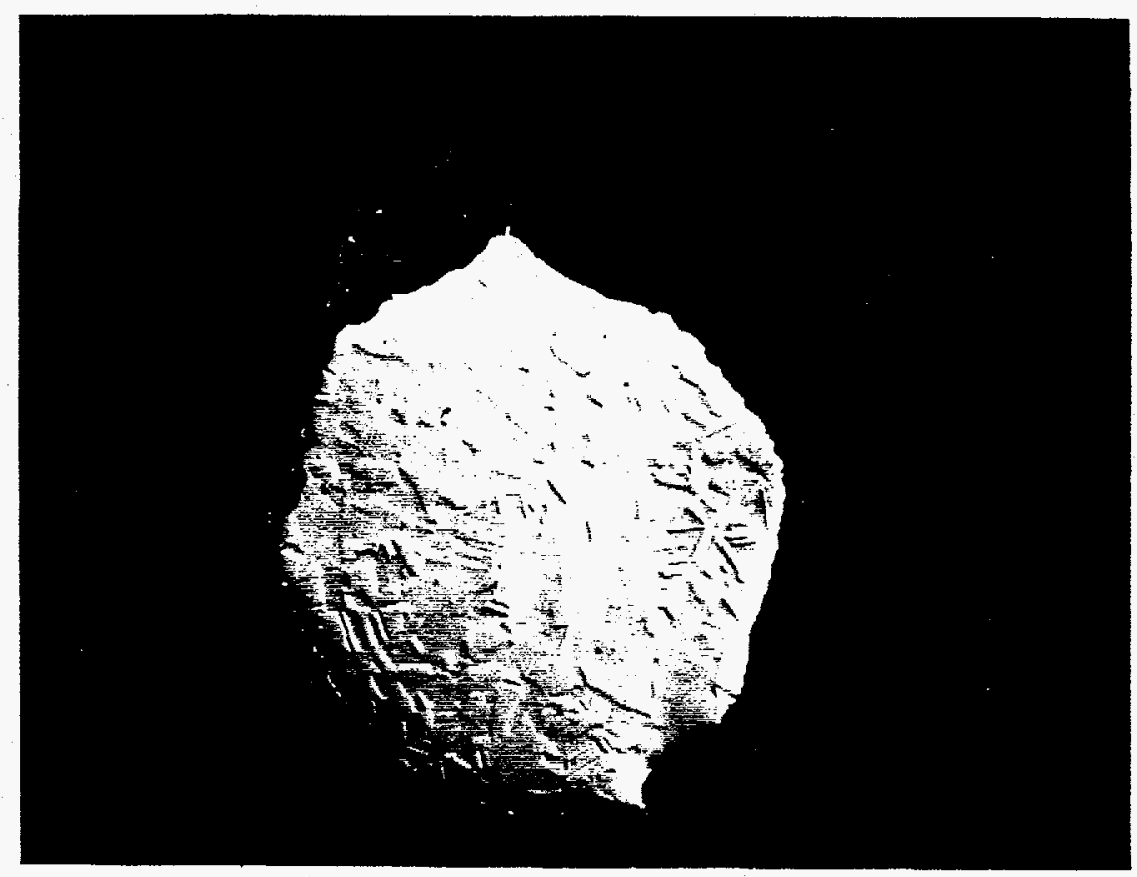

Figure 5

Wire diameter after 40 hours exposure to the reactor between 19.5 and $20 \mathrm{ft}$. (50X as polished)

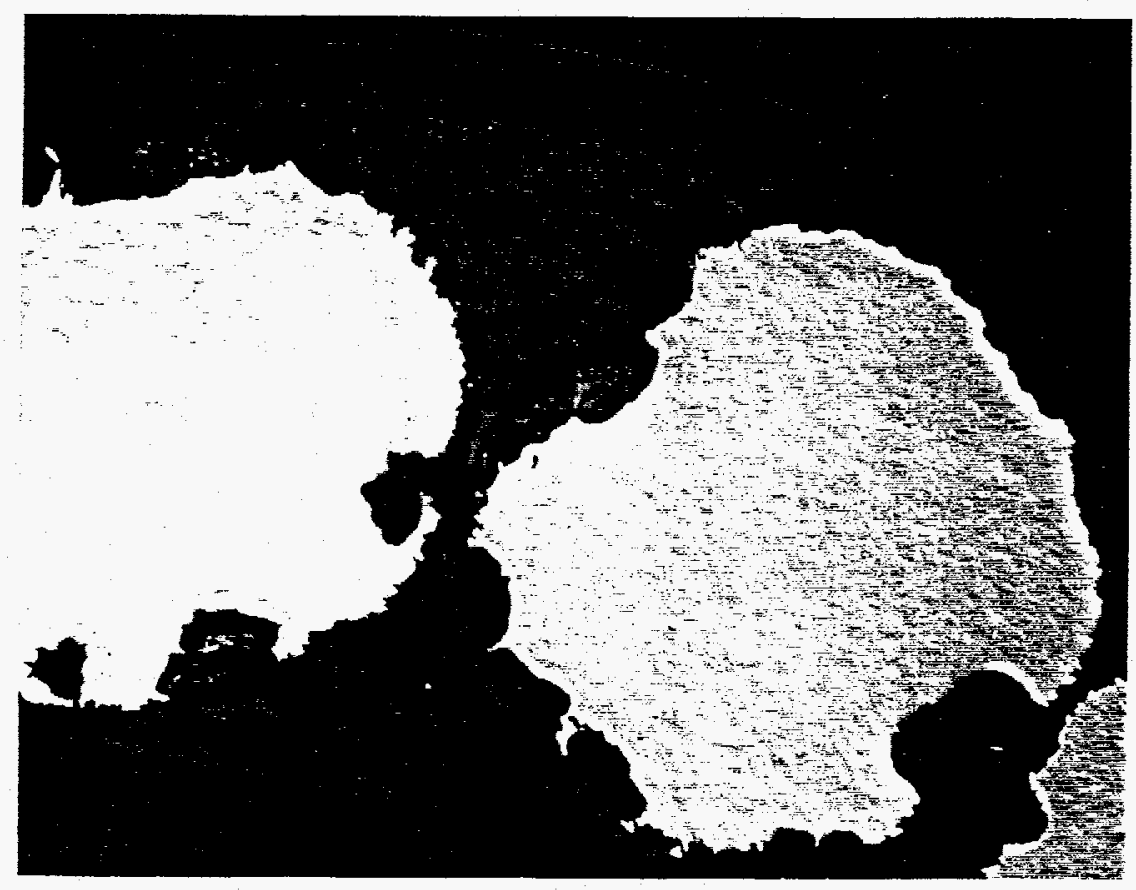

Figure 6

Irregular wire diameter after 20 hours exposure in the reactor between 54 and $54.5 \mathrm{ft}$. (50X as polished) 


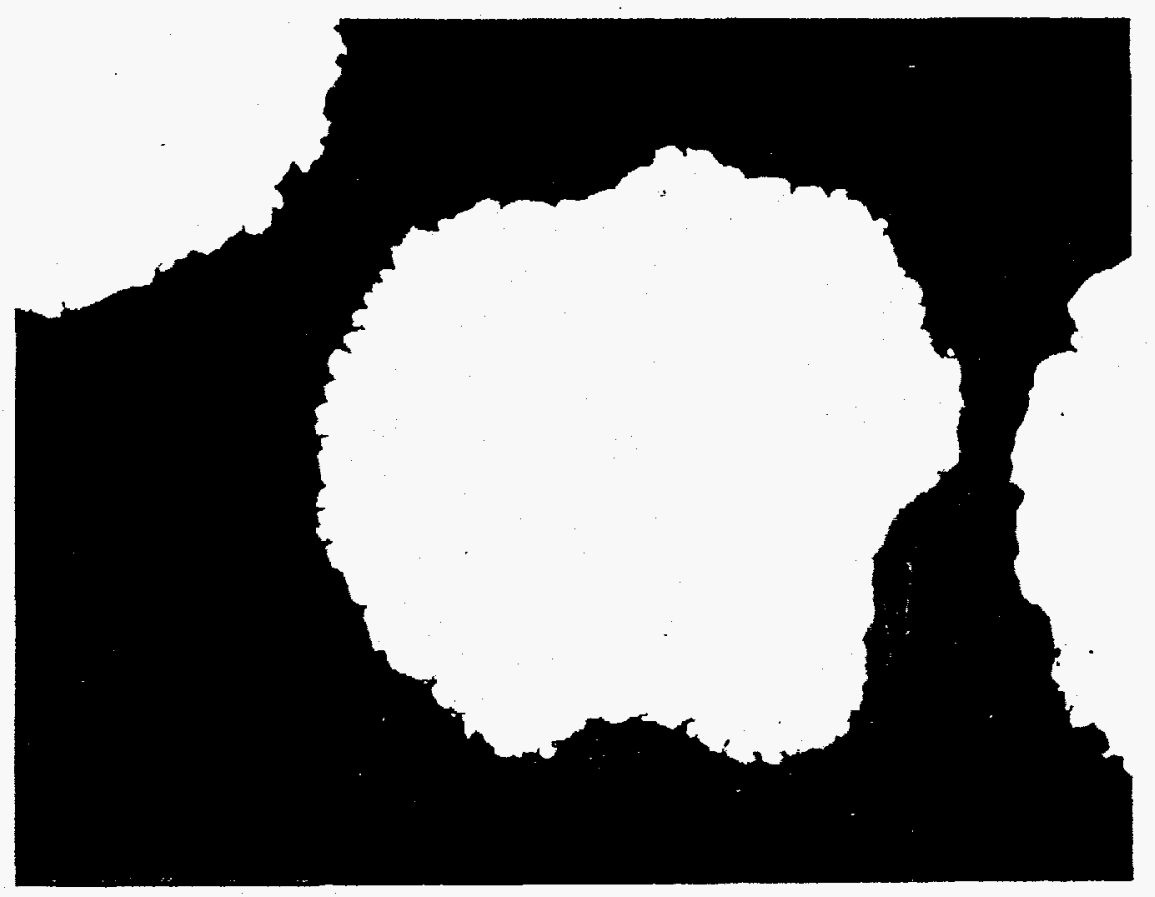

Figure 7

Irregular wire diameter after exposure in the reactor for 40 hours between 19.5 and $20 \mathrm{ft}$. (50X as polished)

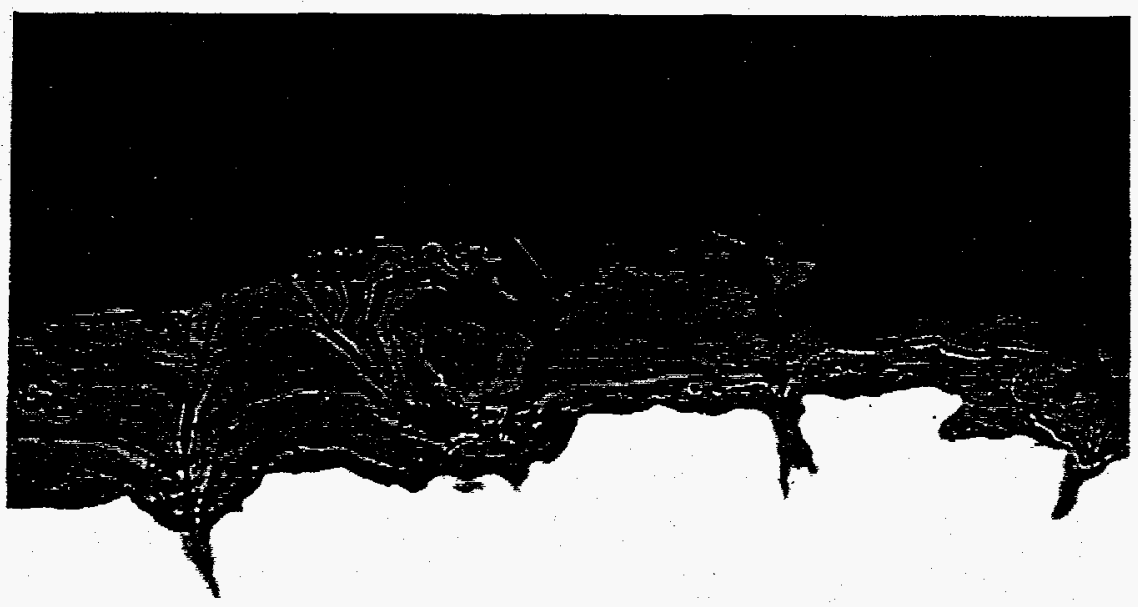

Figure 8

Scale deposit on wire after exposure in the reactor for 40 hours between 19.5 and $20 \mathrm{ft}$. ( $400 \mathrm{X}$ as polished) 


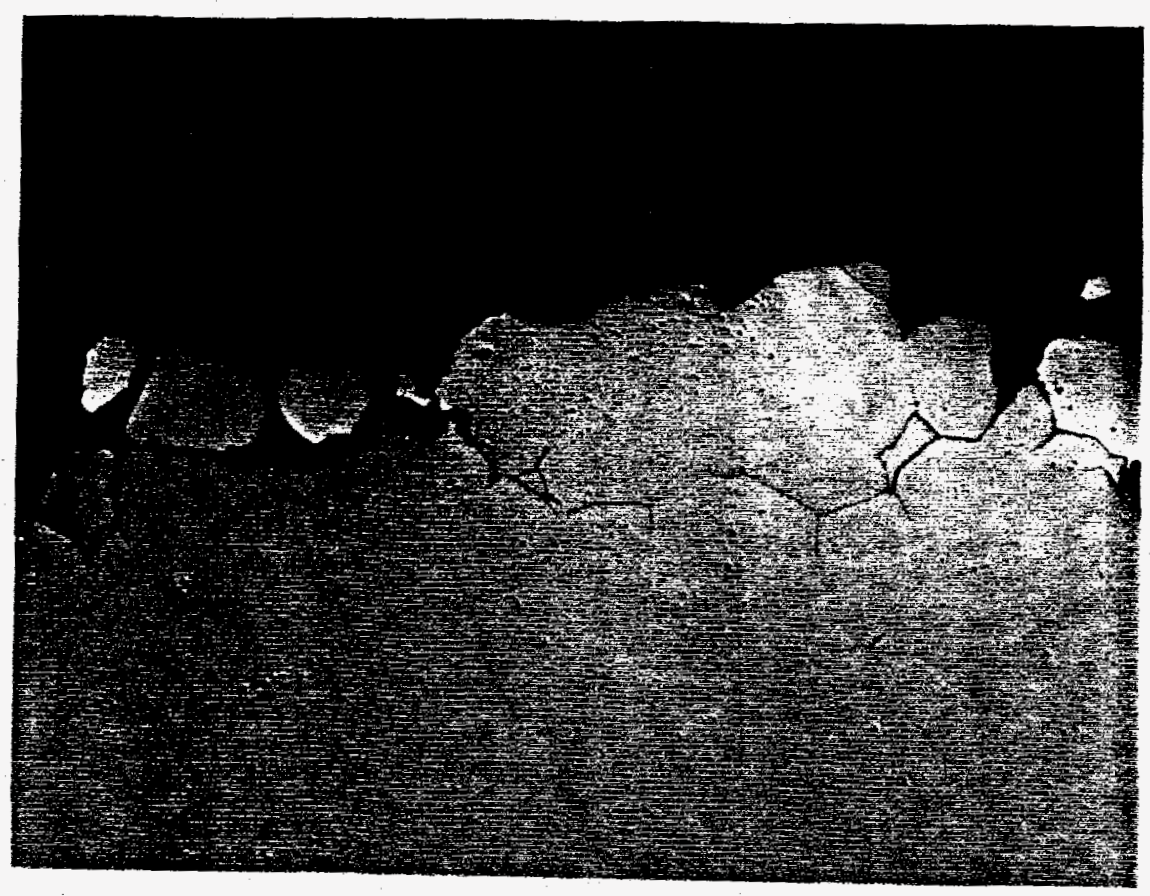

Figure 9

Intergranular attack of wire after exposure in the reactor for 20 hours between 54 and $54.5 \mathrm{ft}$. ( $400 \mathrm{X}$ as polished)

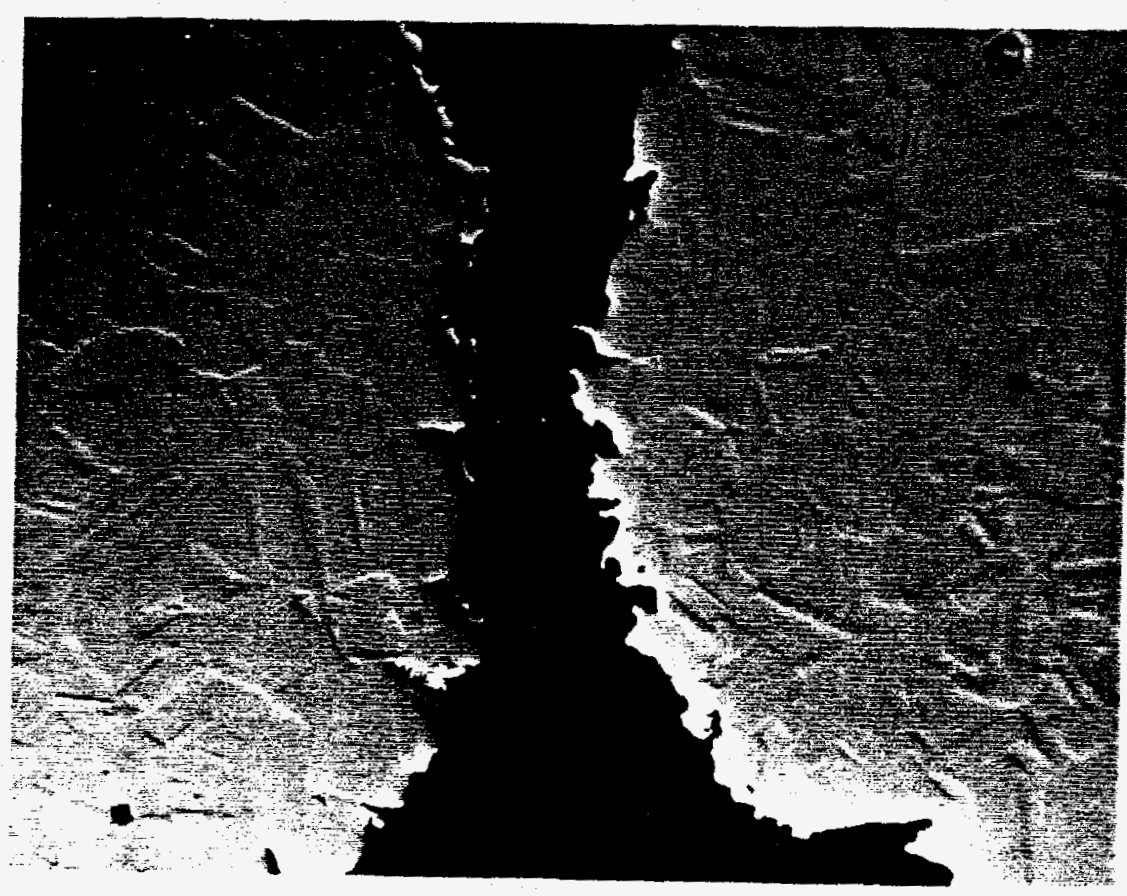

Figure 10

Intergranular attack of wires after exposure in the reactor for 40 hours between 18.5 and $19 \mathrm{ft}$. (100X as polished) 


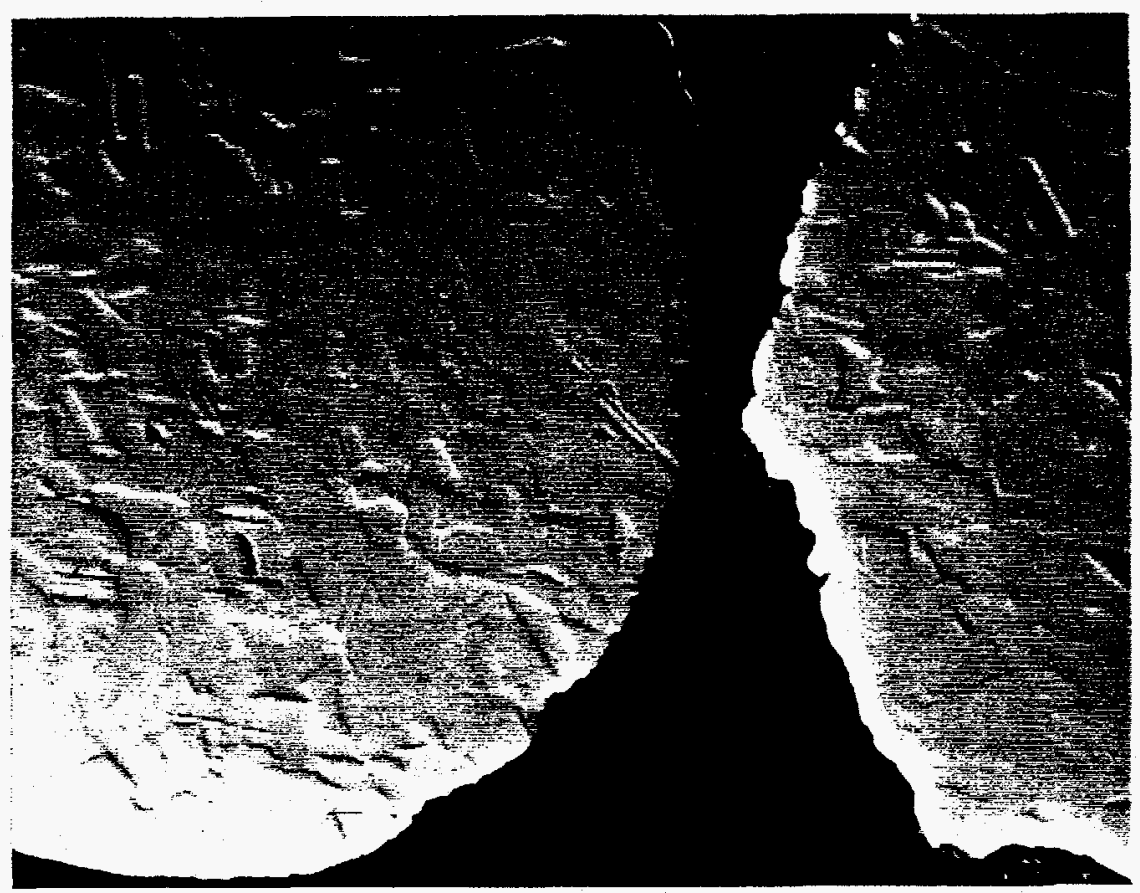

Figure 11

General attack of wires exposed in the reactor for 40 hours between 19 and $19.5 \mathrm{ft}$. (100X as polished)

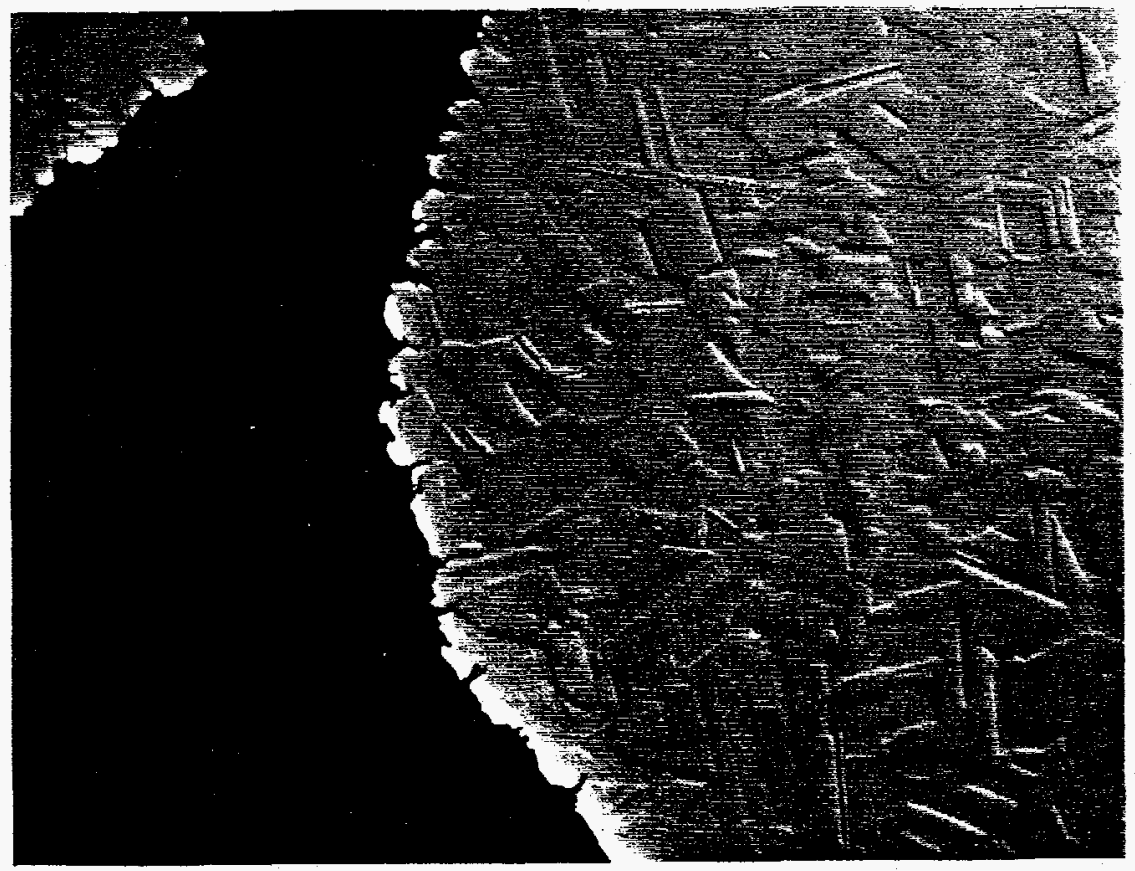

Figure 12

Intergranular attack of wires after exposure in the reactor for 40 hours between 19.5 and $20 \mathrm{ft}$. (100X as polished) 


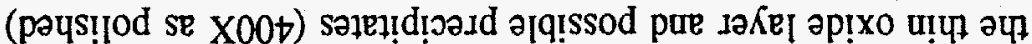

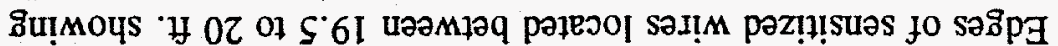

†I ว.กต์!

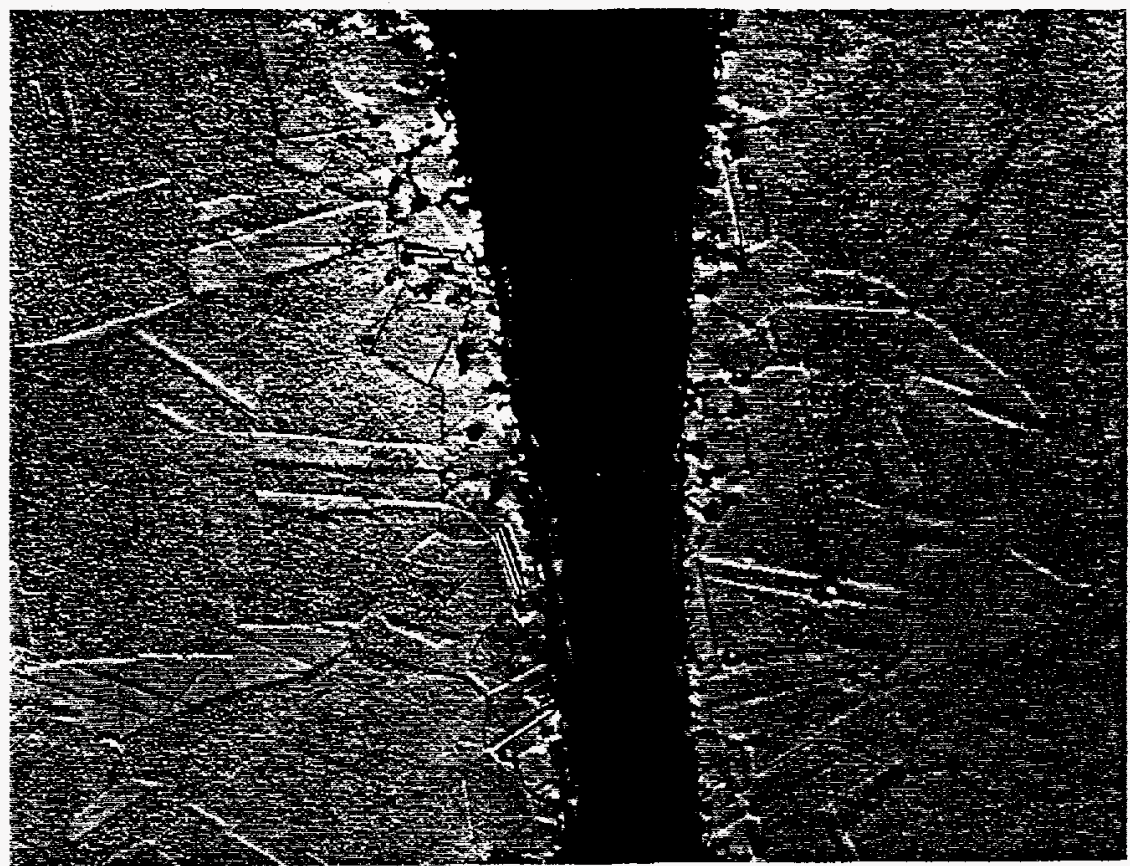

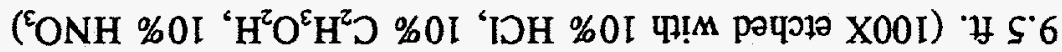
pur 6 чәәмґәq әп!м pəz!!!

EI วIกริ!

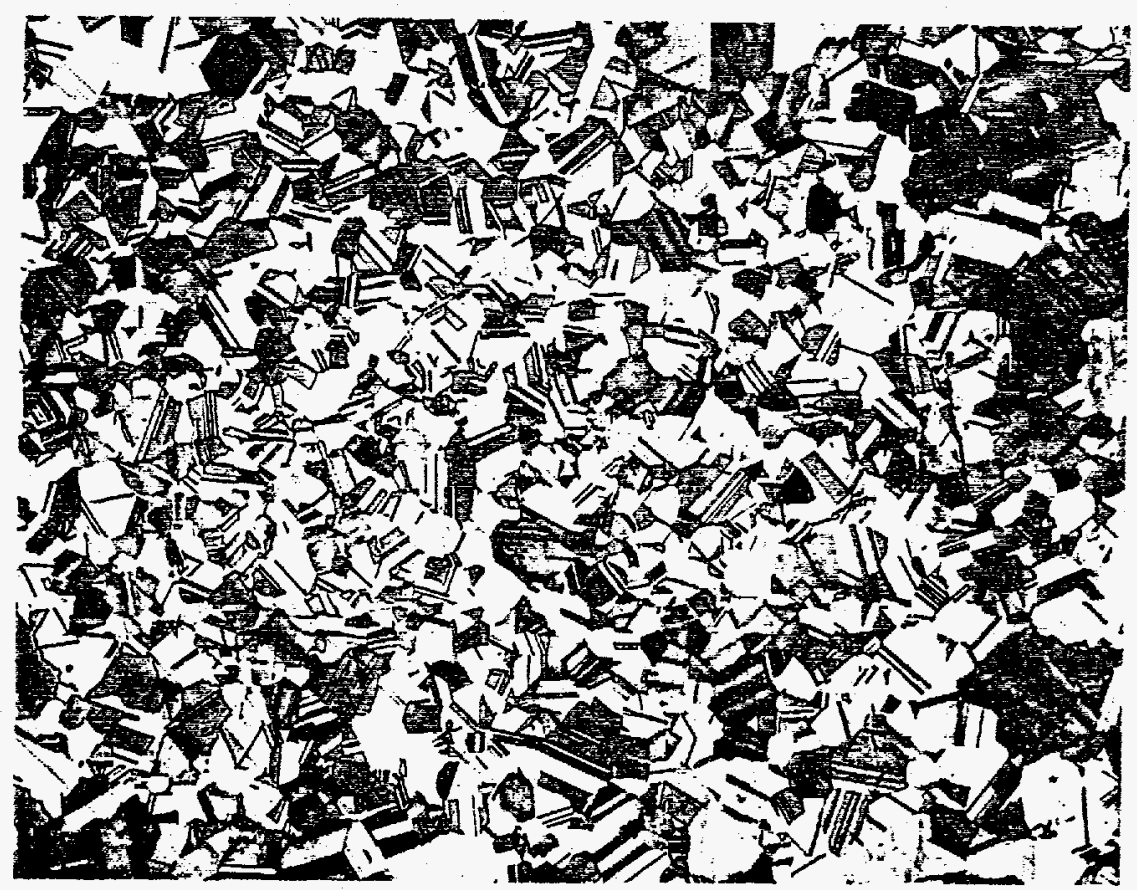




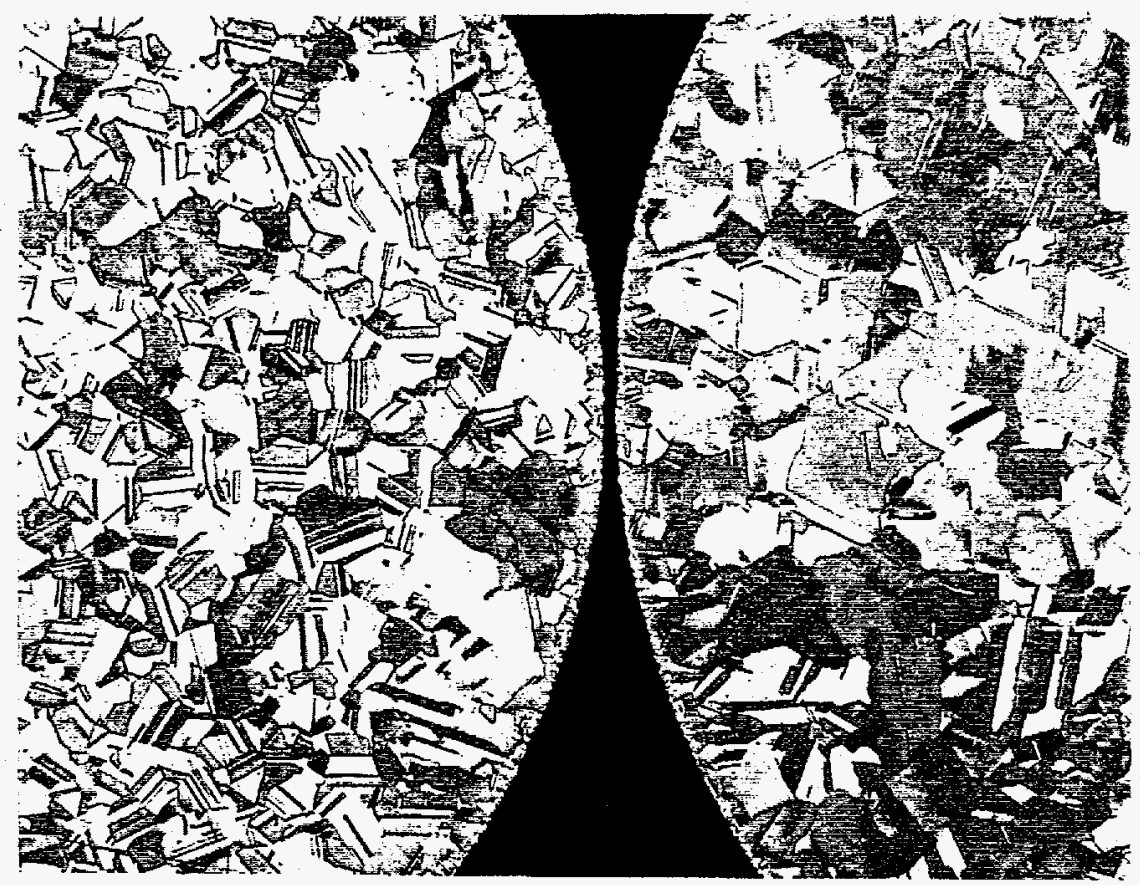

Figure 15

Sensitized wires taken from between 9 and $9.5 \mathrm{ft}$. showing the difference in grain sizes ( $100 \mathrm{X}$ etched with $10 \% \mathrm{HCl}, 10 \% \mathrm{C}_{2} \mathrm{H}_{3} \mathrm{O}_{2} \mathrm{H}, 10 \% \mathrm{HNO}_{3}$ ) 


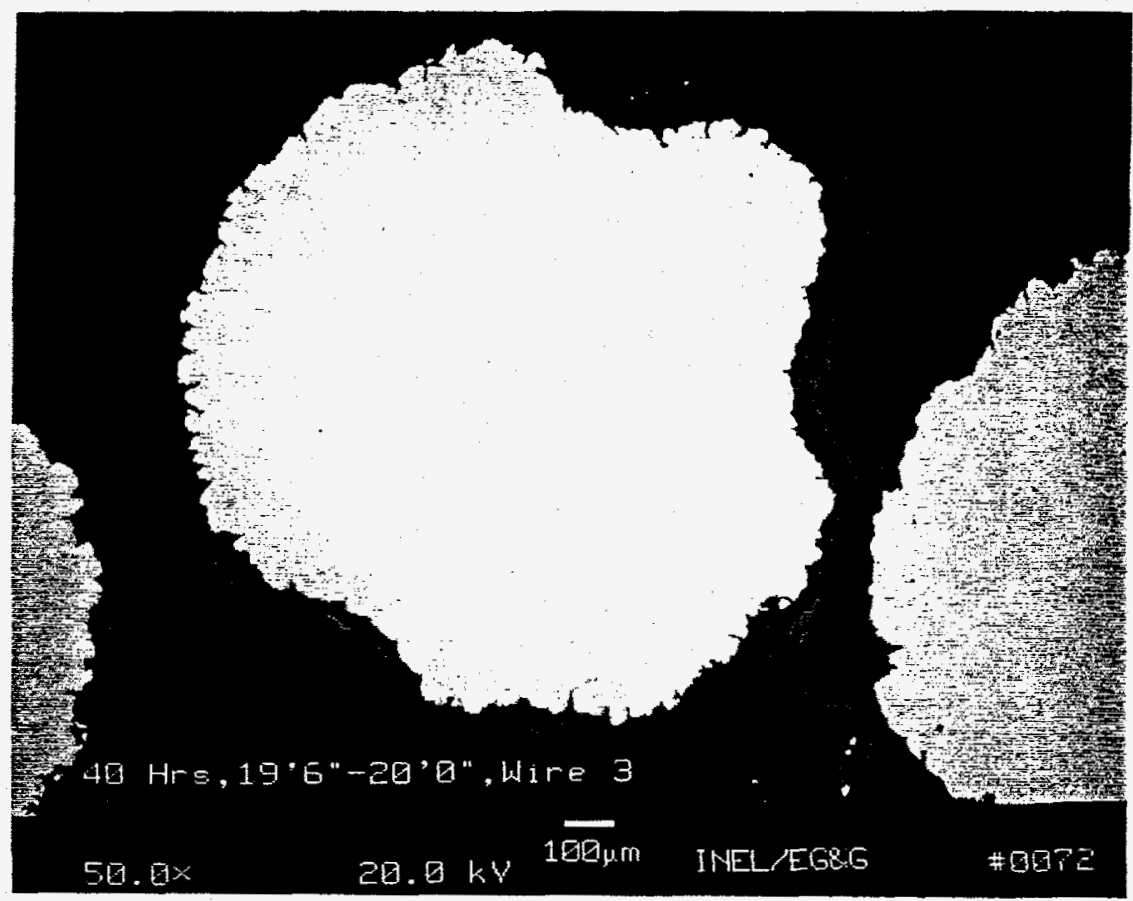

Figure 16

SEM photograph showing scale and intergranular attack

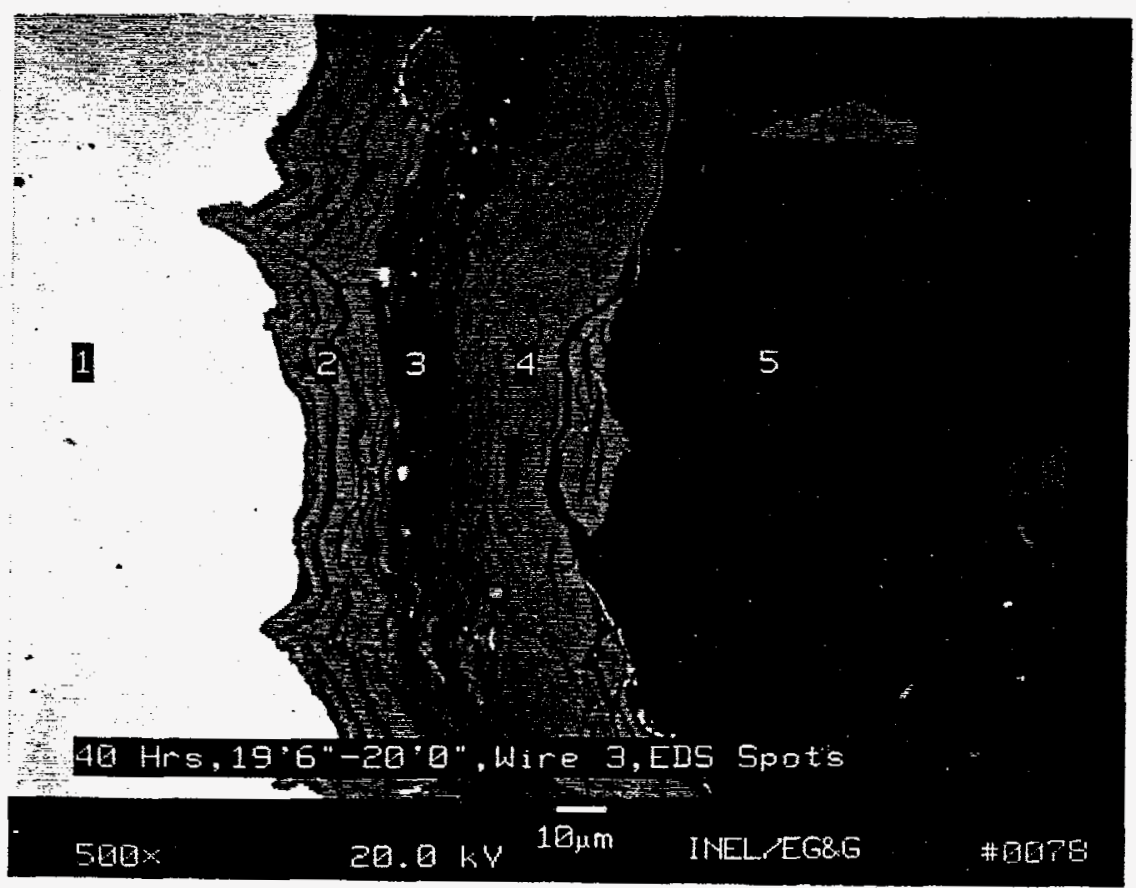

Figure 17

SEM photograph of the wire in Figure 16 at higher magnification, the numbers indicate the location of the spectra where the chemical analysis was taken, spectra 5 is the only location where sulfur was found 


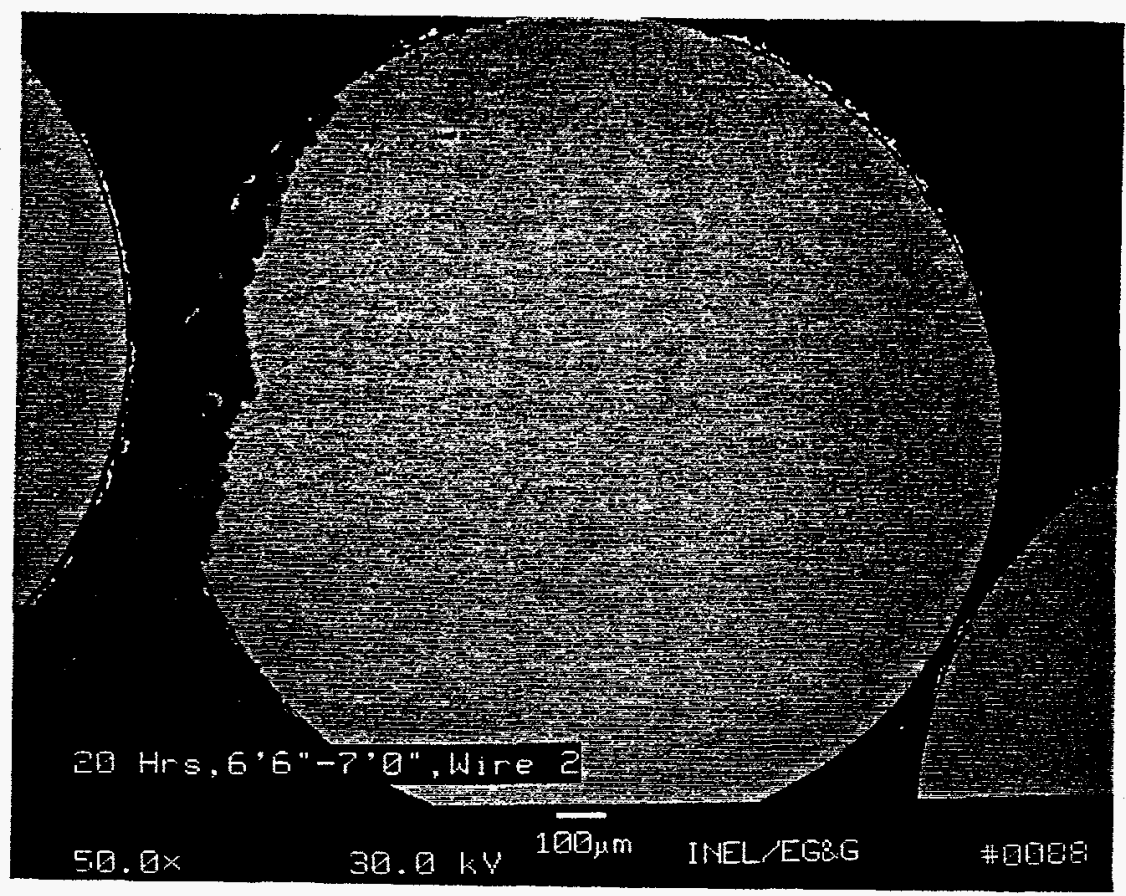

Figure 18

SEM photograph showing large deposit on one side of wire and bright areas on the surface of the wire

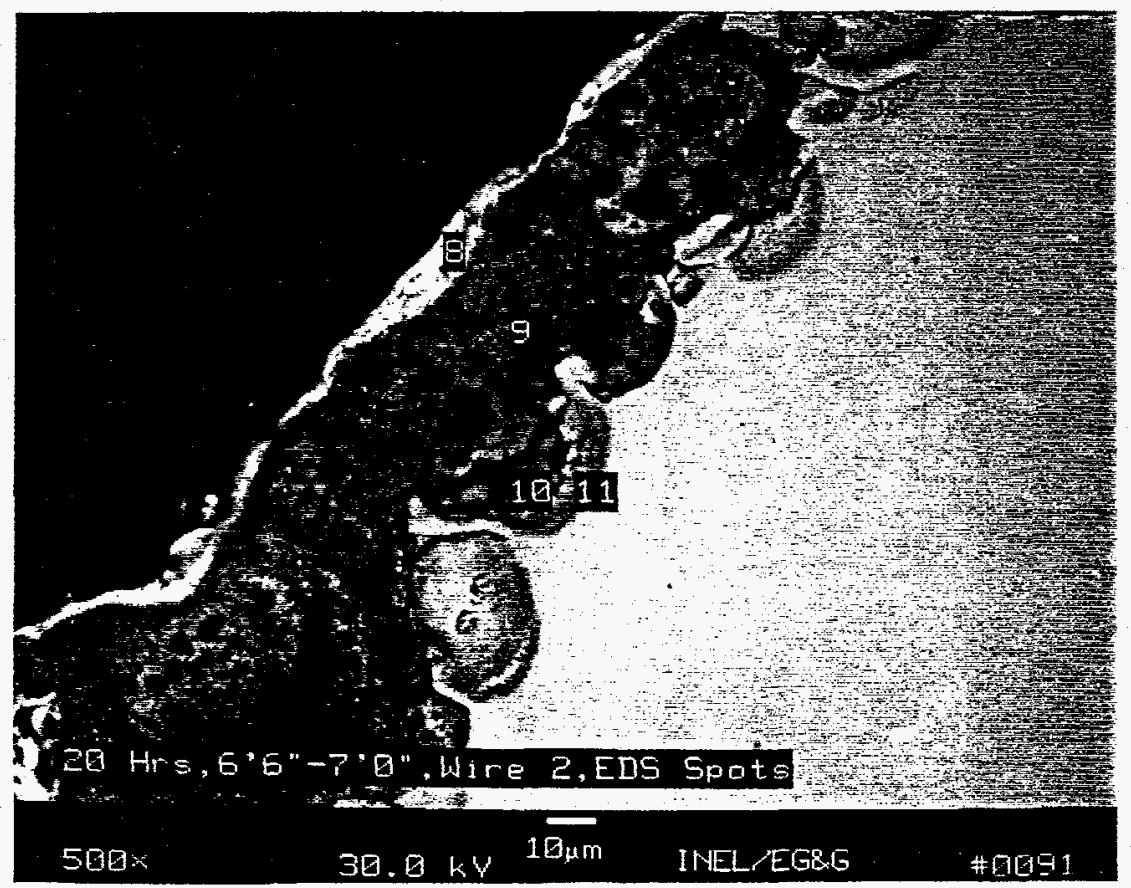

Figure 19

SEM photograph of the scale on the wire in Figure 18 at higher magnification, spectrum 8 is high in $\mathrm{Ce}$ and $\mathrm{Pb}$, the nodule of spectrum 10 is high in $\mathrm{Cl}$ 


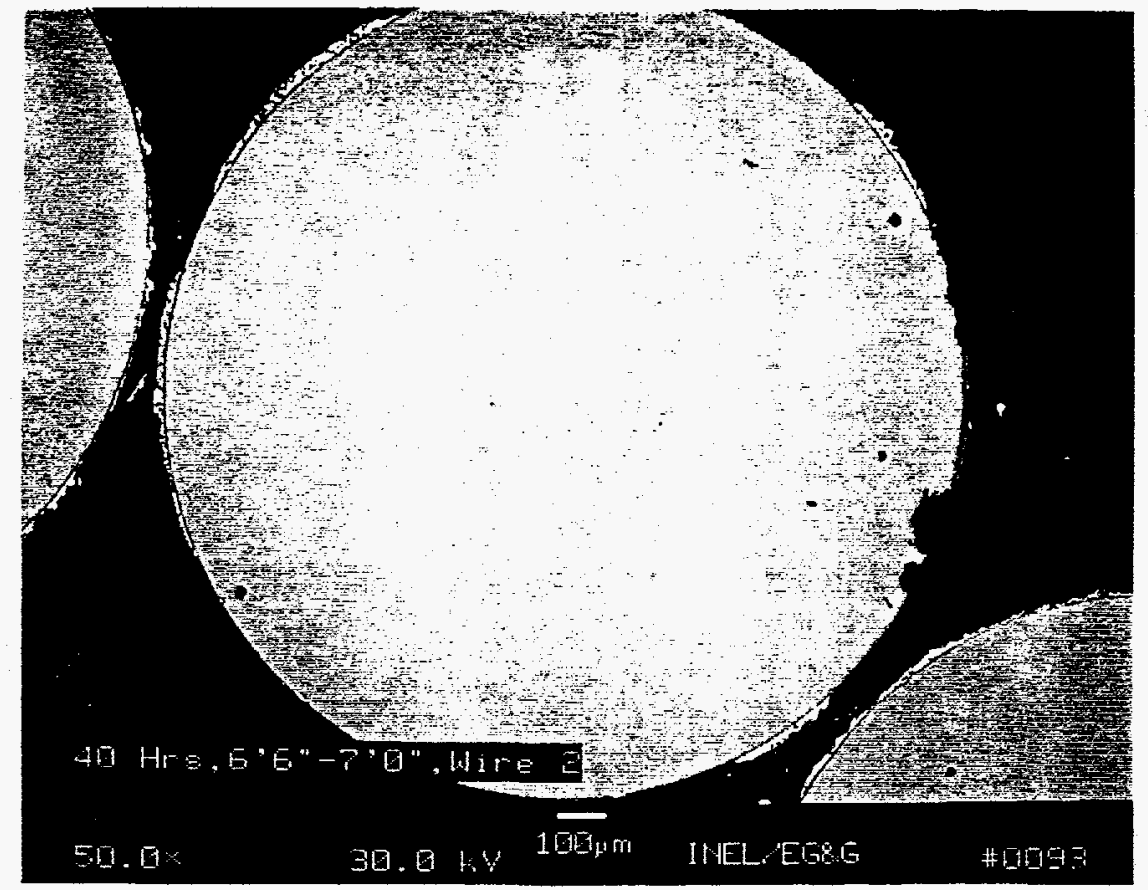

Figure 20

SEM photograph showing bright deposits on the wires

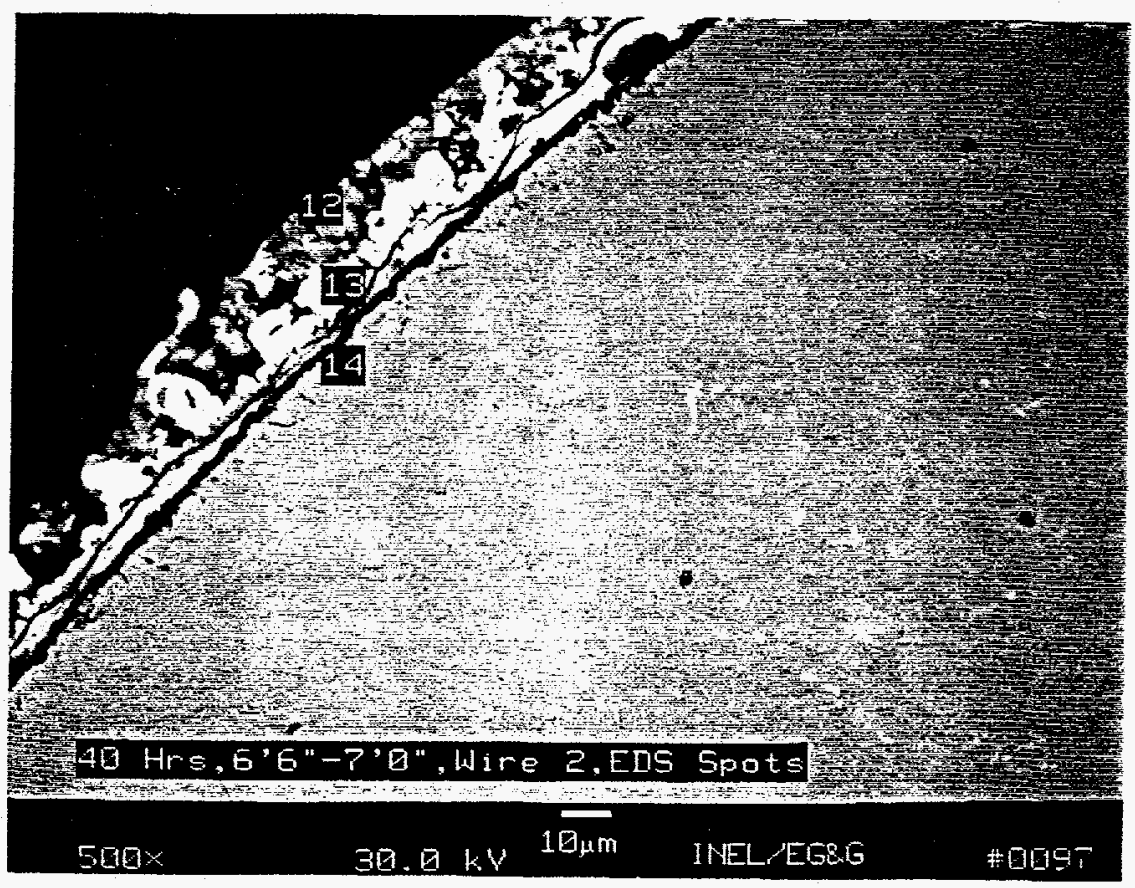

Figure 21

SEM photograph of the bright scale on the wire in Figure 20 at higher magnification, spectra 12 and 13 are high in $\mathrm{Ce}$ and $\mathrm{Pb}$ 


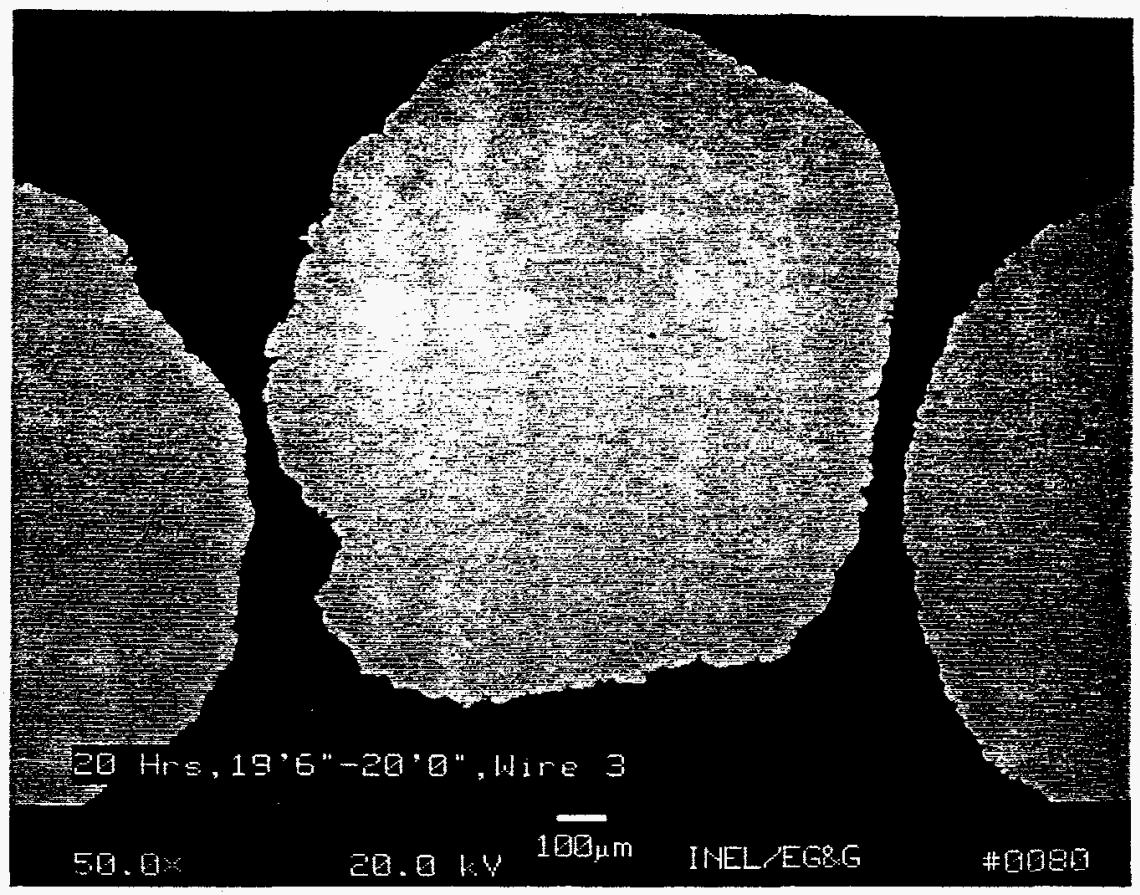

Figure 22

SEM photograph showing dark scale deposit

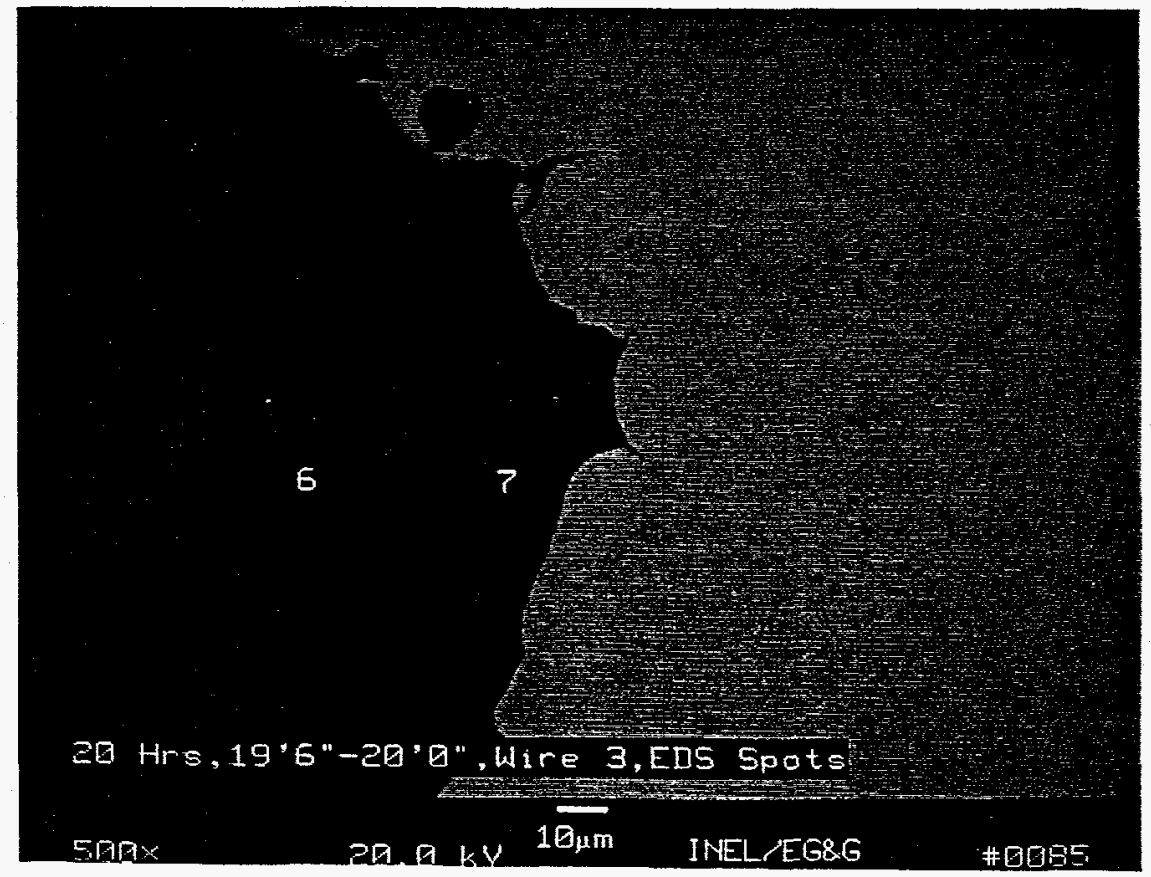

Figure 23

SEM photograph of deposit on the wire in Figure 22 at higher magnification, deposit is mostly oxides of Inconel 625 with some $\mathrm{Ce}$ and $\mathrm{Pb}$ 


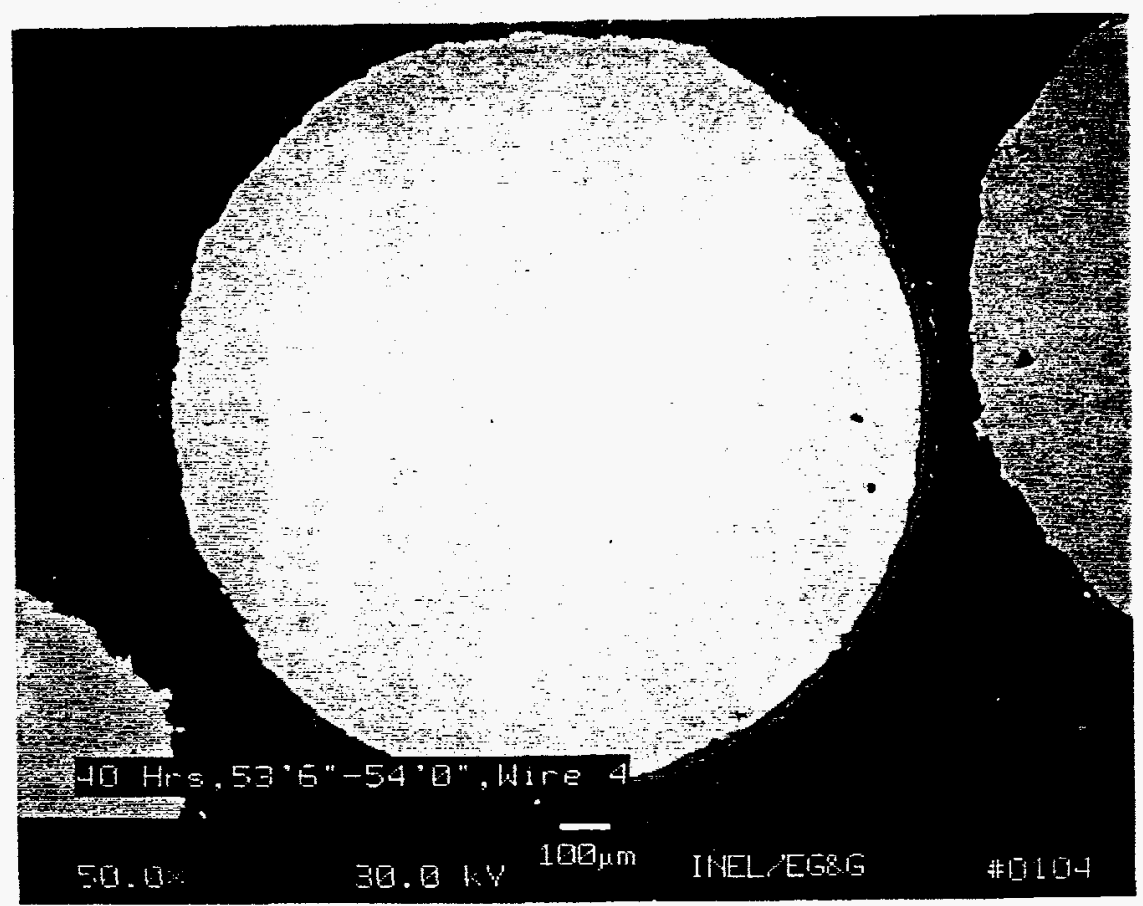

Figure 24

SEM photograph showing continuous deposit around the wire

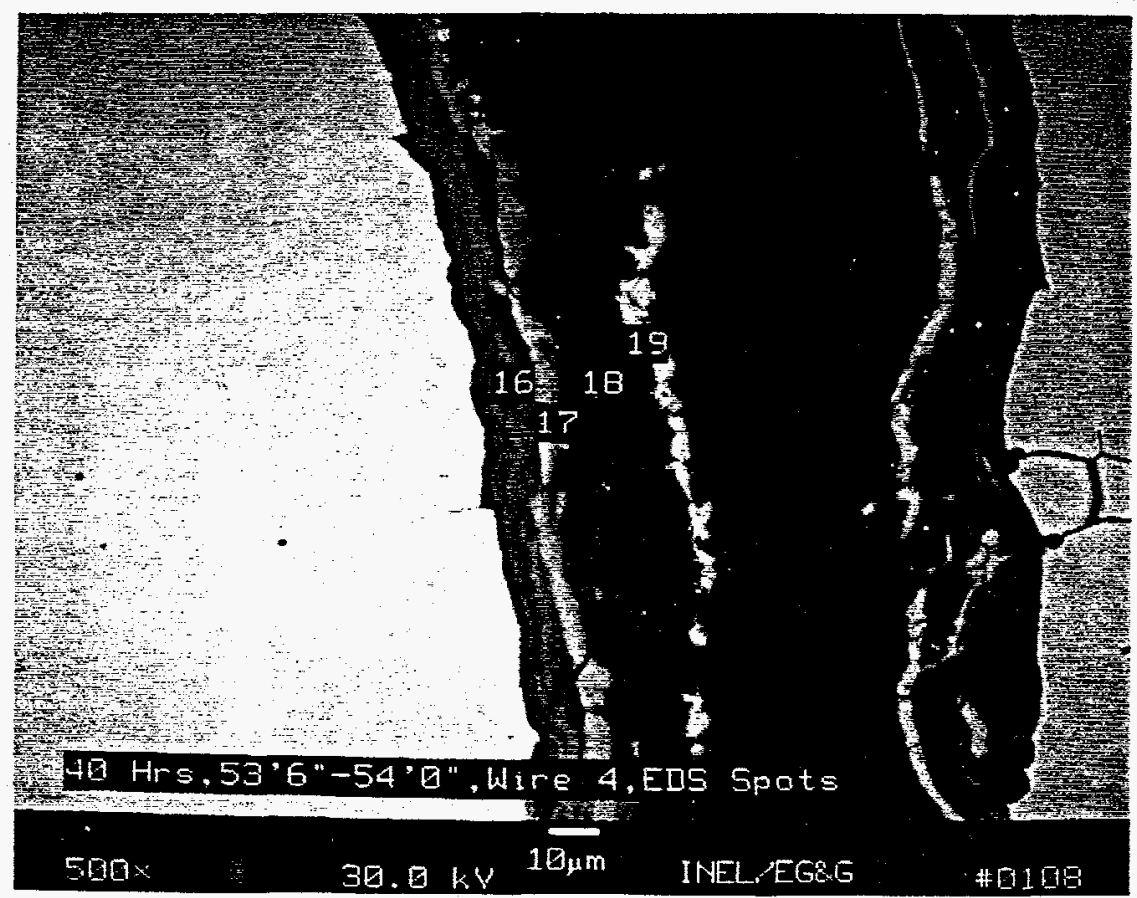

Figure 25

SEM photograph of deposit on the wire in Figure 24 at higher magnification, deposit is high in $\mathrm{Cl}$ and $\mathrm{Zn}$ with $\mathrm{Ce}$ and $\mathrm{Pb}$ present 


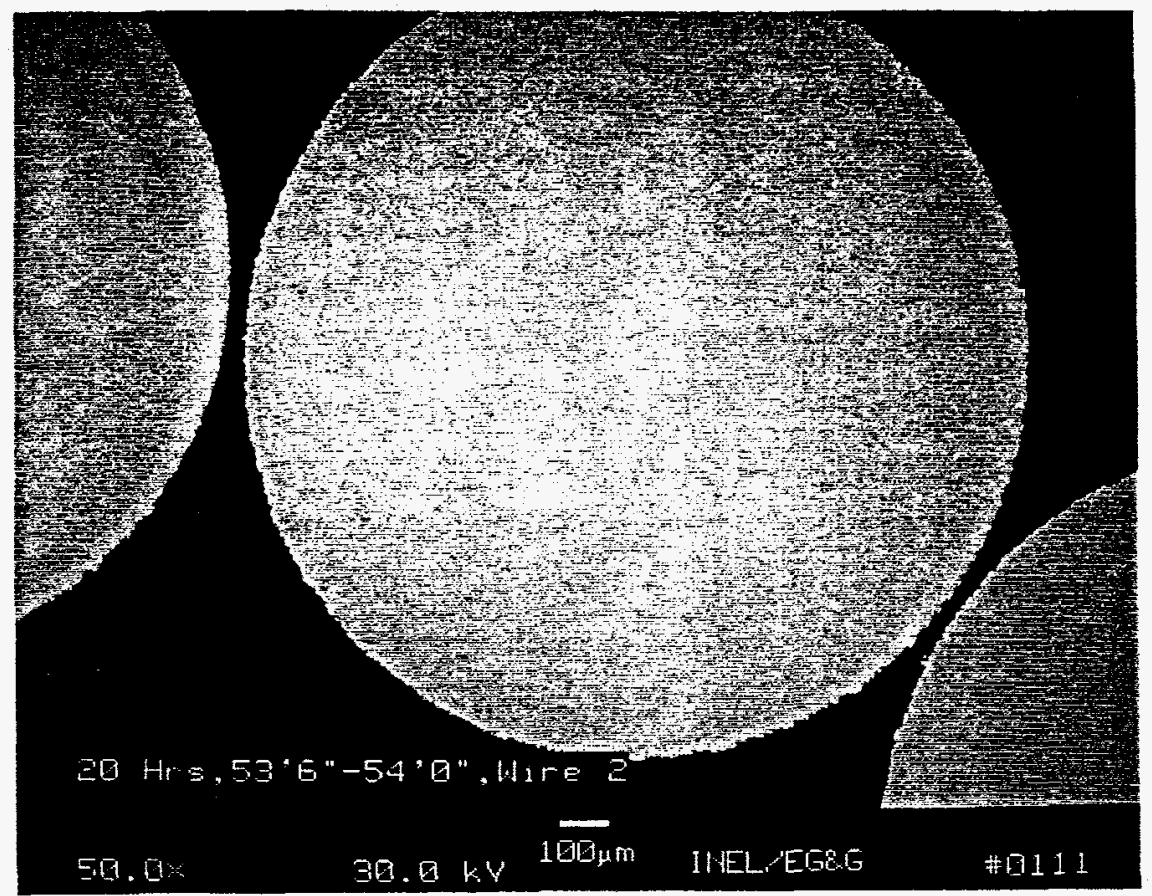

Figure 26

SEM photograph showing little scale deposit

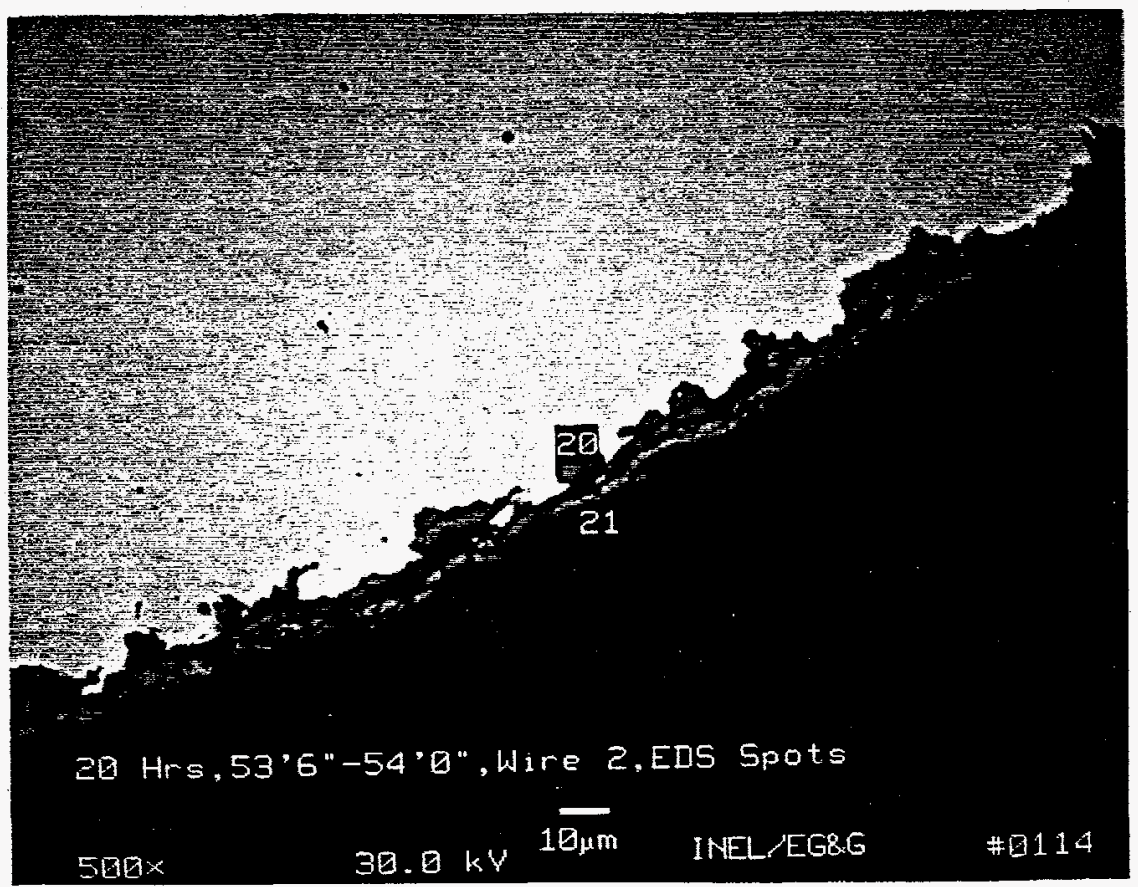

Figure 27

SEM photograph of deposit on the wire in Figure 26 at higher magnification, deposit is oxides of Inconel 625 with $\mathrm{Zn}$ present 


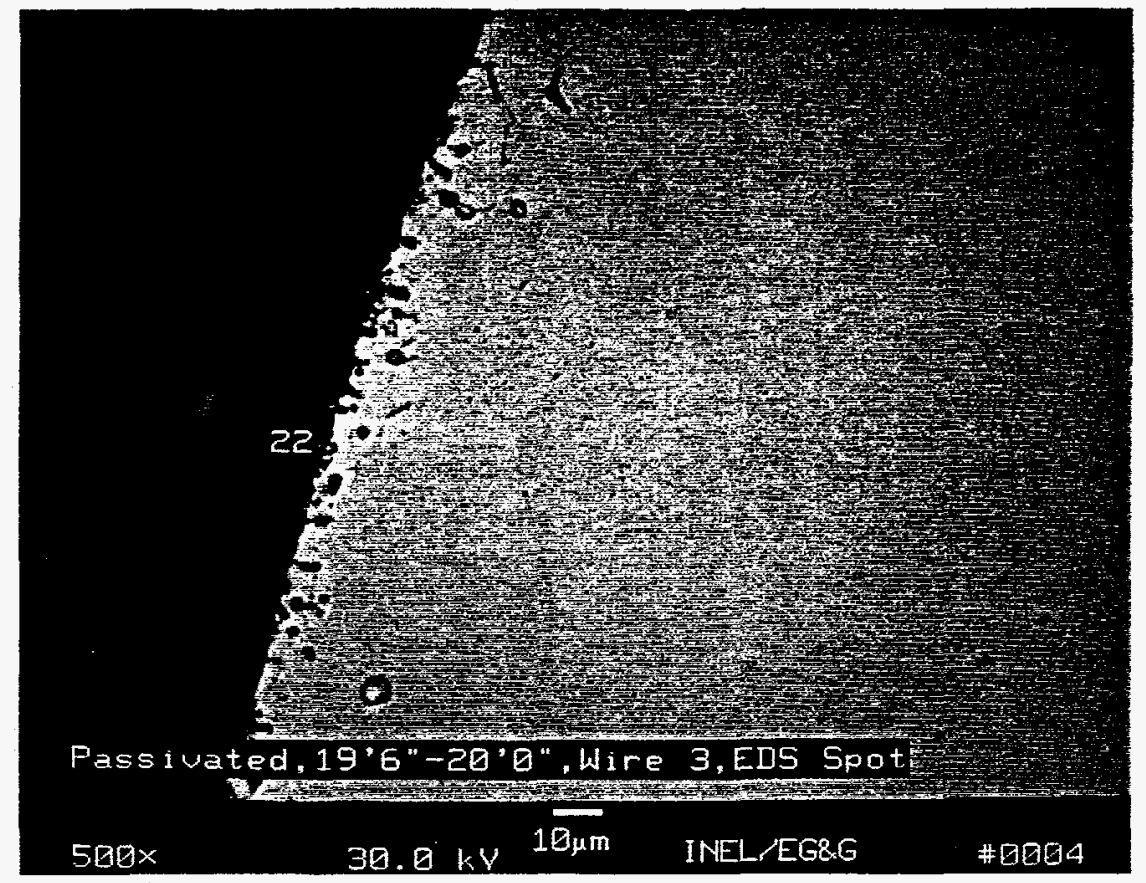

Figure 28

SEM photograph of the edge of the sensitized wire, the oxide layer is high in $\mathrm{Cr}$ (spectrum 22)

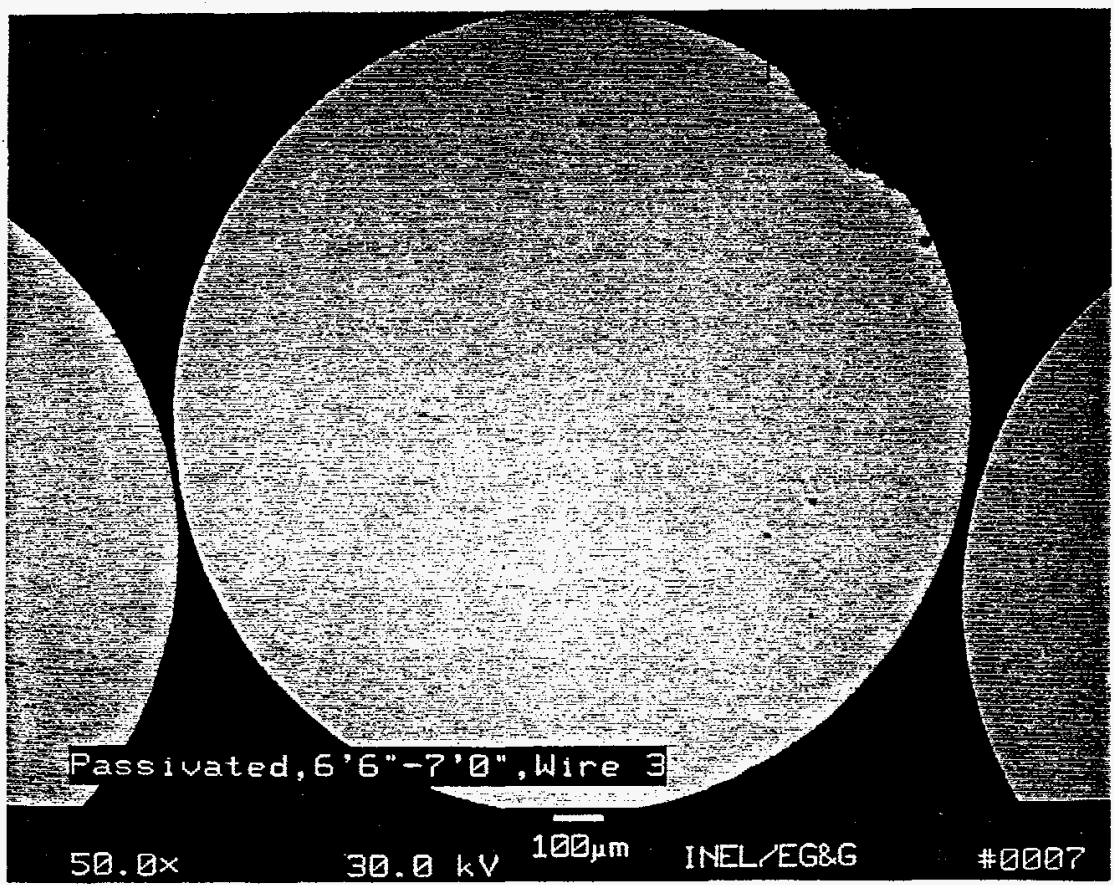

Figure 29

SEM photograph showing large oxide area 


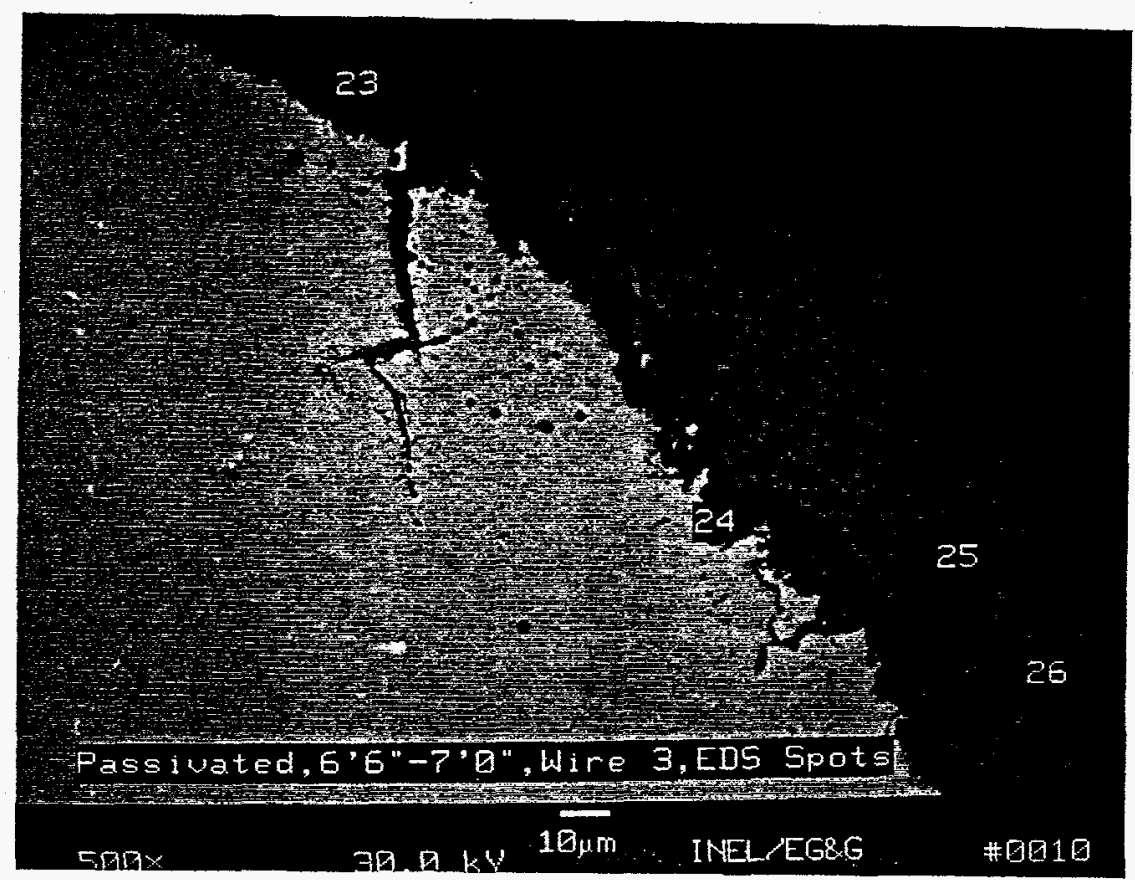

Figure 30

SEM photograph of deposit on the wire in Figure 29 at higher magnification, deposit is oxides of Inconel 625, spectrum 26 is almost entirely nickel oxide

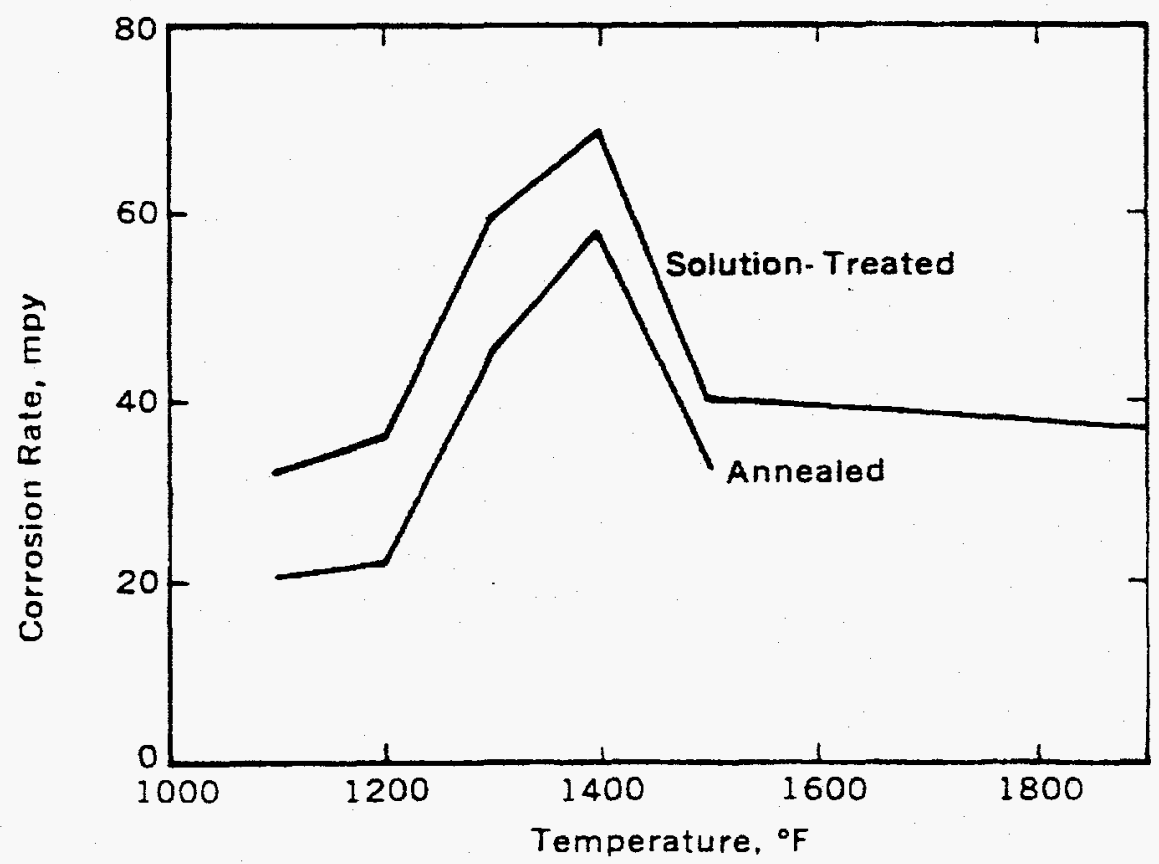

Figure 31

Effect of sensitizing heat treatment on corrosion of Inconel 625 in boiling $65 \% \mathrm{HNO}_{3}$ 


$$
19-\operatorname{Jar}-1993 \quad 14: 41: 27
$$

Accelerating voltage

Beam - sample iriciderice angle

Xray emergerice arigle

Xray - wiridow iriciderice argle
SCWD-1

$30.0 \mathrm{KeV}$

90.0 degrees

35. degrees

Q. Q degrees

STANDARDLESS EDS ANALYSIS
(ZAF CORRECTIONS VIA MAGIC V)

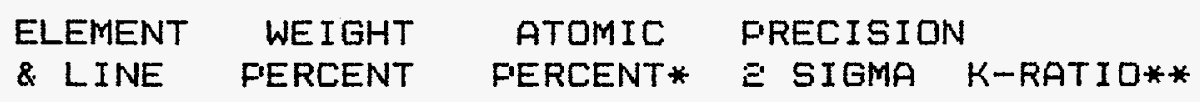

\begin{tabular}{|c|c|c|c|c|}
\hline Al KA & 0. 81 & 1.80 & 0. 15 & 0. \\
\hline$T_{i} K A$ & 0.89 & ㅁ. 11 & 0.014 & ด. QRRB \\
\hline Cr KA & ごコ.ジ & ㄹ.5.59 & D. 27 & Q. $\Xi 16 \square$ \\
\hline Fe KA & $0.8 E$ & $0.8 \theta$ & 0.08 & a. QReB \\
\hline $\mathrm{Ni} \mathrm{KA}$ & $E R .74$ & 61.96 & $0.5 e$ & a. 574 \\
\hline NB KA & 4.37 & E. $8 E$ & 0.78 & a. 035 \\
\hline mo KA & 10.94 & 6.83 & 1.31 & ฉ. 0976 \\
\hline
\end{tabular}

TOTAL

99.99

ITERATIONS

6

*NOTE: ATOMIC FERCENT is rommalized to 100

**NOTE: K-RATIO $=K$ K-RATIO $\times R$

where $R=$ refererice (st andard)/refererice (sample)

NORMALIZATION FACTOR: 1. QRQR 


$$
\text { 19-Jar-1993 } 14: 47: 11
$$

Acceleratirg voltage

Beam - sample iriciderice argle

Xray emergerice arigle

Xray - wiridow incidence angle

STANDARDLESS EDS ANALYSIS

(ZAF CORRECTIONS VIA MAGIC $V$ )

\section{ELEMENT \\ \& LINE}

WEIGHT
$K-R A T I O * *$ FERCENT

TOTAL
๑. 01732

Q. $018=4$

D. 3337

Q. Q Q

Q. 0.0735

0. 0770

D. 1141

Mo KA

口 *

AI KA

Cr KA

Fe $K A$

$N i K A$

NG KA

.

0.93
$0 . .37$
37.17
$0 . .77$
8.30
8.76
13.35
30.95

30.95
SCWO-E

30. Q KeV

90.0 degrees

35.0 degrees

Q. Q degrees

\section{FRECISION \\ Z SIGMA}
ㅁ. 12
Q. 0.5
D. 30
D. DE
‥ 18
‥ $8 E$
1.15
‥ 18
Q. $8 E$
1.15
‥ 18
Q. $8 E$
1.15

FORMULA

Aleaz
TiQE
Creos
Fed
NiO
NoEdS
MaO3

MaOS
DXIDE

FERCENT

100.000

\title{
* determined ey stoichidMetry
}

ITERATIONS 20

**NOTE: K-RATID $=K$ K-RATIO $\times R$

where $R=$ refererice (standard)/reference (sample)

\author{
NORMALIZATION FACTOR: 1.89e
}




$$
\text { 19-Jari-1993 } 14: 50: 14
$$

Accelerating voltage

Beam - sample iriciderice argle

Xray emergerice angle

Xray - wirdow incidence argle
SCWO-3

$30.0 \mathrm{KeV}$

90. degrees

35. 1 degrees

Q. D. degrees

STANDARDLESS EDS ANALYSIS

(ZAF CORRECTIONS VIA MAGIC $V$ )

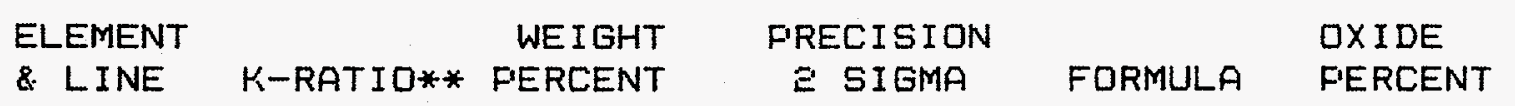

\begin{tabular}{|c|c|c|c|c|c|c|}
\hline Al & $K A$ & Q. $\nabla_{1} \square_{1}$ & 3.96 & 0.32 & $A 1 \Xi \square \Xi$ & 7.49 \\
\hline$T_{i}$ & $K A$ & 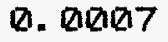 & 0.07 & 0.03 & TiOE & 0. 12 \\
\hline $\mathrm{Cr}$ & $K A$ & Q. 1147 & $11 . B Q$ & 0. 22 & CrEOJ & 17. 25 \\
\hline $\mathrm{Fe}$ & $K A$ & ด. ฉロモB & D. 27 & 0.07 & $F \in O$ & 0. 35 \\
\hline $\mathrm{Ni}$ & KA & D. 4577 & $48.4 B$ & $0.5 \mathrm{e}$ & $\mathrm{NiO}$ & 61.69 \\
\hline Nb & KA & Q. $\square \Xi E \square$ & 3.74 & 0.75 & NbPas & 5.35 \\
\hline$M=$ & $\begin{array}{l}\text { KA } \\
*\end{array}$ & 0.0434 & $\begin{array}{r}5.17 \\
=6.50\end{array}$ & 1.04 & $\operatorname{mo0} 3$ & 7 \\
\hline
\end{tabular}

TOTAL

99.99

* DETERMINED BY STOICHIOMETRY

ITERATIONS 7

**NOTE: K-RATIO $=K$-RATIO $\times R$

where $R=$ refererice (standard)/refererice (sample)

NORMALIZATION FACTOR: 1.889 


$$
\text { 19-Jar-1993 14:53:30 }
$$

Accelerating voltage

Eearn - sample inciderice argle

Xray emergerice angle

Xray - wirdaw iriciderice argle
SCWO-4

30. $0 \mathrm{KEV}$

90. Degrees

35.0 degrees

Q. Degrees

STANDARDLESS EDS ANALYSIS

(ZAF CORRECTIONS VIA MAGIC $V$ )

\section{ELEMENT \\ \&. LINE}

$$
\begin{array}{r}
\text { WEIGHT } \\
K-\text { RATIO** FERCENT }
\end{array}
$$

จ. จดeอ

a. QRะE5

ถ. 4167

Q. 0141

จ. 0302

๑. 0688

0.0773

MO $K A$

Q *

TOTAL
9.78

31.22

45.63

1. 77

3. 48

7. 89

31. $2 \mathrm{E}$

\section{PRECISION \\ ? SIGMA}

ט. 11

0. 0.5

Q. 34

Q. 10

D. 14

Q. 87

1. 10
FORMULA

Alea3

TIDE

Creos

$F \in O$

$\mathrm{NiO}$

NBEOS

$\mathrm{MoO} 3$
OXIDE

PERCENT

1.25

Q. 45

6E. 69

ㄹ. 28

4. 43

11. 29

13. $6 \mathrm{E}$

180.280

* Determined ey stoIChiometry

ITERATIONS ea

**NOTE : K-RATIO $=K$-RATIO $\times R$ where $R=$ refererice (staridard)/refererice (sample)

NORMALIZATION FACTOR: 1.891 


$$
\text { 19-Jar-1993 } 14: 56: \Xi 0 \quad \text { SCWD-5 }
$$

$$
\begin{array}{lc}
\text { Accelerating voltage } & 30.0 \mathrm{KeV} \\
\text { Beam - sample inciderice angle } & 90.0 \text { degrees } \\
\text { Xray emergence angle } & 35.0 \text { degrees } \\
\text { Xray - window inciderice angle } & 0.0 \text { degrees }
\end{array}
$$

\begin{tabular}{|c|c|c|c|c|c|}
\hline ELEMENT & $K-$ ROT I $\Pi^{*} *$ & WE I GHT & FRECISION & 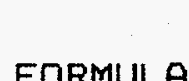 & $\begin{array}{l}\text { QXIDE } \\
\text { FERCENT }\end{array}$ \\
\hline$K A$ & 0.1383 & $\Xi \not .9 \square$ & ㅁ. 24 & 503 & 5こี. 19 \\
\hline $\mathrm{Cr} \quad \mathrm{KA}$ & a. 0398 & 4. 18 & a. 12 & Crea3 & E. 12 \\
\hline$F e K A$ & D. DQROL 4 & 0.014 & D. Q1E & FeO & D. 05 \\
\hline $\mathrm{Ni} \mathrm{KA}$ & D. 3118 & Зコ.7こ & 0. 36 & NiO & 41.64 \\
\hline $0 \quad *$ & & 4 Ė. 15 & & & \\
\hline
\end{tabular}

STANDARDLESS EDS ANALYSIS

(ZAF CORRECTIONS VIA MAGIC $V$ )

TOTAL

99.99

* determined ey stoichiometry

ITERATIONS ZQ

**NOTE: K-RATIO $=K$-RATIO $\times R$

where $R$ = refererice (standard)/refererice (sample)

NORMALIZATION FACTOR: $=.441$ 
19-Jar-1993 15: $210: 013$

Accelerating voltage

Beam - sample inciderice argle

Xray emergence arigle

Xray - wiridow inciderice arigle

STANDARDLESS EDS ANALYSIS

(ZAF CORRECTIONS VIA MAGIC $V$ )

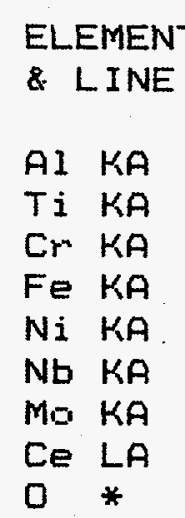

TOTAL
30. $\mathrm{KEV}$

90. degrees

35.0 degrees

Q. degrees

* DETERMINEd BY STOICHIOMETRY ITERATIONS ZQ

**NOTE: K-RATIO $=K$ K-RATIO $\times$ R

where $R=$ reference (standard)/reference (sample)

NORMALIZATION FACTOR: 1.892 


$$
19-J a r_{1}-1993 \quad 15: 08: 36
$$

Accelerating voltage

Beam - sample iriciderice argle

Xray emergence arigle

Xray - wiridow iriciderice arigle

STANDARDLESS EDS ANALYSIS

(ZAF CORRECTIONS VIA MAGIC $V$ )
3Q. $0 \mathrm{KEV}$

90. 0 degrees

35. 1 degrees

Q. de degrees

ELEMENT
\& LINE
AI KA
CI KA
Ti KA
Cr $K A$
Ni $K A$
Nb $K A$
MO KA
Fb LA
D *

TOTAL

\begin{abstract}
WEIGHT K-RATIO** FERCENT
\end{abstract}

\begin{abstract}
D. QR⿴囗十
\end{abstract}
Q. 01833

จ. จดะอง

D. 3357

0. 17394

a. 0864

D. $12 E 3$

a. DURE

‥ 45

D. 58

B. 34

37.89

4.45

9.77

14. 71

0. 85

\author{
PRECISION \\ E SIGMA
}

30.96
0.10
Q. 07
Q. 0.5
Q. 33
จ. 16
D. 89
1. 26
Q. 18

FORMULA

OXIDE

FERCENT
Q. 86

0. 58

Q. 57

55.38

5. 66

13. 97

ออ. 017

จ. 91

100.010

* DETERMINED EY STOICHIOMETRY

ITERATIONS EQ

**NOTE: K-RATIO $=K$-RATIO $\times R$

where $R=$ refererice (staridard)/refererice (sample)

NORMALIZATION FACTOR: 1.890 


$$
\text { 19-Jar-1993 15:15:07 }
$$

Accelerating voltage

Beam - sample iriciderice angle

Xray emergerice angle

Xray - wiridow iriciderice angle

STANDARDLESS EDS ANALYSIS

(ZAF CORRECTIONS VIA MAGIC $V$ )

\section{ELEMENT}

\& LINE

WEIGHT
K-RATIO** FERCENT

จ.

Al $K A$

Cl $\mathrm{KA}$

Cr KA

$\mathrm{Ni} K A$

No KA

Mo $K A$

Ce LA

PB LA

口 *

‥ 0761

0.0182

Q.

๑. QE9B

จ. 3674

a. 1408

TOTAL

18.57
$30.0 \mathrm{KeV}$

30. Q degrees

35.0 degrees

a. a degrees

* determined by staichiometry

I TERATIONS

Ea

FRECISION
e SIGMA

Q. 13

ㅁ. 13

D. 24

D. 15

Q. 69

1. 33

0. 50

a. 6.8
QXIDE

FORMULA

FERCENT

AlE03

$\mathrm{Cl}$

Creos

$\mathrm{NiO}$

NDPOS

MoO3

CeEa3

Fbo
1. 르

1. 03

1อ. 61

E. $E E$

4. $\Xi 9$

10. 97

49. 34

17. $9 \Xi$

* NOTE: K-RATIO $=K$ K-RATIO $\times$ R

where $R$ = refererice (staridard)/refererice (sample)

NORMALIZATION FACTOR: 1.887 


$$
19-J a r_{1}-1993 \quad 15: 18: 50
$$

Acceleratirg voltage

Eeam - sample iriciderice argle

Xray emergence arigle

Xray - wiridow iriciderice argle

STANDARDLESS EDS ANALYSIS

(ZAF CORRECTIONS VIA MAGIC $U$ )

\section{ELEMENT}

\& LINE

Al $K A$

Cl $K A$

$T i K A$

$\mathrm{Cr} K A$

Fe KA

$N i K A$

Nb KA

$M G K A$

o *

TOTAL
30. $0 \mathrm{KeV}$

90. Degrees

35. 0 degrees

Q. Degrees
WEIGHT

K-RATIQ** FERCENT

จ. 0.056

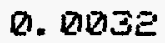

Q. 0038

a. $\Xi 976$

0. D1065

0. 1015

Q. 1123

D. 0756

R. 0756

.

1. 66

D. 56

D. 43

33.11

D. 78

11. 38

12. 86

8. $8 E$

30.35

\author{
FRECISION \\ E SIGMA
}
0. 06
D. 015
Q. 27
Q. 07
Q. 20
D. 91
0. 98

Q. 13 A1203

CI

Tide

CrEOZ

FeO

$\mathrm{NiO}$

NbEoS

$\mathrm{MoO} 3$
OXIDE

FERCENT

3. 14

b. 56

‥ 72

48. 39

1. 00

14. 48

18. 40

13.30

* Determined BY STOICHIDMETRY

ITERATIONS EQ

**NOTE: K-RATIO $=K$-RATIO $\times R$

where $\mathrm{R}=$ refererice (staridard)/reference (sample)

NORMALIZATION FACTOR: 1. 199E 


$$
\text { 19-Jan-1993 15: Eะ:31 SCWO-10 }
$$

$$
\begin{array}{lc}
\text { Accelerating voltage } & 30.0 \mathrm{KeV} \\
\text { Eeam - sample iriciderice angle } & 90.0 \text { degrees } \\
\text { Xray emergerice angle } & 35.0 \text { degrees } \\
\text { Xray - window iriciderice arigle } & 0.0 \text { degrees }
\end{array}
$$

STANDARDLESS EDS ANALYSIS

(ZAF CORRECTIONS VIA MAGIC V)

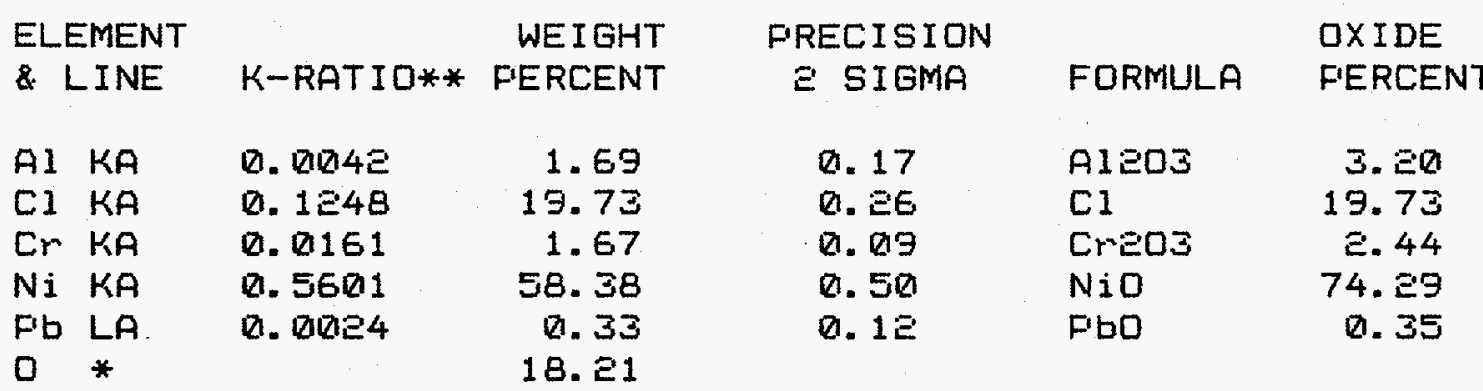

TOTAL

1010. Q121

* DETERMINED BY STOICHIOMETRY

ITERATIONS $\quad 7$

**NOTE: K-RATIO $=K$ K-RATIO $\times R$

where $R=$ reference (staridard)/refererice (sample)

NORMALIZATION FACTOR: $1.89 \square$ 


$$
\text { 19-Jar-1993 15: :7: घอ }
$$

Acceleratirg voltage

Bean - sample inciderice arigle

Xray emergerice arigle

Xray - window iriciderice argle

STANDARDLESS EDS ANALYSIS

(ZAF CORRECTIONS VIA MAGIC $V$ )

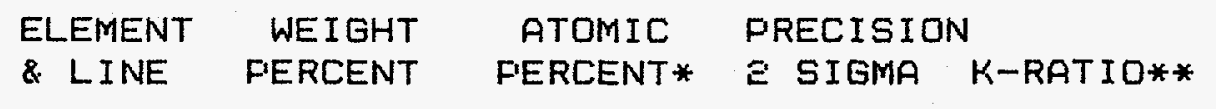
AI KA
$7.3 E$
14.77
1. 13
ט. 27
0. 11
0. 0153
Cl $K A$
Q. 74
3. 71
B. 18
Q. 01039
Cr KA
3.73
ㅁ. 31
D. 05
D. DIBBE
Fe KA
83.48
77.38
D. 49
4. 42
2. 50
1. 00
0. 01039
D. 8217
Q. 0.385

TOTAL 100.01

\section{TERATIDNS}

7

*NOTE: ATOMIC FERCENT is nommalized to 1 QR

**NOTE: K-RATIO $=K$-RATIO $\times R$

where $R=$ reference (staridard)/reference (sample)

NORMALIZATION FACTOR: 1. QRQR 
19-Jar-199コ 15: อョ:17

Acceleratirg voltage

Beam - sample inciderice argle

Xray emergerice angle

Xray - window inciderice argle

STANDARDLESS EDS ANALYSIS

( $Z A F$ CORRECTIONS VIA MAGIC $V$ )

\begin{abstract}
ELEMENT
\&. LINE

$\mathrm{Cr} K A$

Fe $K A$

$\mathrm{Ni} K A$

CE LA

F'b LA

口 *
\end{abstract}

K-RATIO**

WEIGHT

PERCENT

Q. 0110

0. 01010

Q. 81057

D. 6654

0. 8965

TOTAL

* determined by staichiometry

ITERATIONS

EQ
SCWD-1E

30. $\mathrm{KeV}$

3Q. 0 degrees

35.0 degrees

0. (1)grees

**NOTE: K-RATIO $=K$-RATIO $\times$ R

where $R=$ reference (staridard)/reference (sample)

NORMALIZATION FACTOR: 1.459 


$$
\text { 19-Jar-1993 15: Зこ:4E }
$$

Accelerating voltage

Beam - sample iriciderice argle

Xray emergence angle

Xray - window iriciderice argle
SCWO-13

30. Q KeV

90. Degrees

35.7 degrees

Q. 2 degrees

STANDARDLESS EDS ANALYSIS

(ZAF CORRECTIONS VIA MAGIC $V$ )

\begin{abstract}
ELEMENT
\& LINE

Cr KA

$F \in K A$

Ni $K A$

Ce LA

$\mathrm{Fb}$ LA

o *
\end{abstract}

TOTAL
WEIGHT K-RATIO** FERCENT
D. 0254

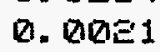
0. 01090
Q. 6440
D. 1038
Е. 73
Q. 27
1.05
E9. 38
12. 13
14. 44

\section{Z SIGMA \\ 0.22
0.10
0.15
0.68
0.69}

FRECISION
QXIDE

FORMULA FERCENT

CrEaz
FeO
Nid
CeEOJ
Pbo

3.99

Q2. 35

1.33

B1. $E$ 'E

13. 817

\title{
* DETERMINED BY STOICHIOMETRY I TERATIONS 20
}

**NOTE: K-RATIO $=K$-RATIO $\times R$ where $R$ = reference (staridard)/refererice (sample)

\author{
NORMALIZATION FACTOR: $\quad 1.458$
}


$19-\operatorname{Jar}-1993 \quad 15: 36: 34$

Accelerating voltage

Beam - sample incidence angle

Xray emergerice arigle

Xray - window inciderice arigle
SCWO-14

30. $0 \mathrm{KeV}$

30. degrees

35.0 degrees

Q. degrees

STANDARDLESS EDS ANALYSIS

(ZAF CORRECTIONS VIA MAGIC $V$ )

\begin{tabular}{lccll} 
ELEMENT & \multicolumn{1}{c}{ WEIGHT } & PRECISION & & OXIDE \\
\& LINE & K-RATIO** FERCENT & E SIGMA & FORMULA & FERCENT
\end{tabular}

\begin{tabular}{|c|c|c|c|c|c|c|}
\hline Al & $K A$ & 0.0154 & 5.15 & 0.21 & A1203 & 9.74 \\
\hline$T_{i}$ & $K A$ & Q. QREJ & 0.65 & D. DUE & TiDE & 1. 018 \\
\hline $\mathrm{Cr}$ & $K A$ & 0.3640 & 38.50 & 0.30 & CrEDJ & 5E. $\Xi 7$ \\
\hline $\mathrm{Fe}$ & $K A$ & 0. $\cos 30$ & $a .3 E$ & 0.013 & $\mathrm{FeO}$ & 0.46 \\
\hline $\mathrm{Ni}$ & $K A$ & D. $1 E \otimes E$ & 18.18 & Q.ZE & NiD & E3. 14 \\
\hline Nb & $K A$ & 0.0135 & 1.58 & 0.43 & NbEOS & $\Xi . \Xi \epsilon$ \\
\hline Mo & $K A$ & D. $\Delta \geq 59$ & 3.189 & 0.79 & $\mathrm{MaD3}$ & 4.64 \\
\hline $\mathrm{Ce}$ & LA & Q. 0152 & 1. 56 & Q. $1 \Xi$ & Cecas & 1.83 \\
\hline Pb & LA & 0.01043 & 0.56 & $0 . \Xi 0$ & Fbo & Q. $G Q$ \\
\hline 0 & * & & 30.38 & & & \\
\hline
\end{tabular}

TOTAL

1010. 01

* DETERMINED BY STOICHIDMETRY

ITERATIONS ZQ

**NOTE: $K$-RATIO $=K$-RATIO $\times R$

where $R=$ refererice (standard)/reference (sample)

NORMALIZATION FACTOR: 1.890 


$$
19-\operatorname{Jar}-1933 \quad 15: 39: 08
$$

Accelerating voltage

Beam - sample iriciderice argle

Xray emergerice angle

Xray - wiridow inciderice argle

STANDARDLESS EDS ANALYSIS

( $Z$ AF CORRECTIONS VIA MAGIC $V$ )
ELEMENT

\& LINE

K-RATIO** FERCENT

A1 $K A$

Ti KA

Cr KA

$\mathrm{Fe} K A$

$N i K A$

Nb KA

Mo KA

0 *

TOTAL
$30.0 \mathrm{KeV}$

90.0 degrees

35.7 degrees

Q. Degrees
ถ. DIRES
D. 78
Q. Quen
Q. 24
‥ 3162
Q. Quage
35.94
Q. Q
จ. 0709
D. 77
2. 45
ㅁ. 1726
7.94
20. 188
31.80

PRECISIO
E SIGM
0.13
0.05
0.35
0.019
0.14
0.94
1.58

OXIDE

FORMULA

FERCENT

* DETERMINED eYY STOICHIOMETRY

ITERATIONS EQ

**NOTE: K-RATIO = K-RATIO $* R$

where $R=$ reference (staridard)/reference (sample)

NORMALIZATION FACTOR: 1.891 


$$
\text { 19-Jar-1993 } 15: 41: 42 \quad \text { SCWO-16 }
$$

$$
\begin{array}{lc}
\text { Accelerating voltage } & 30.0 \text { KeV } \\
\text { Beam - sample iriciderice arigle } & 90.0 \text { degrees } \\
\text { Xray emergence angle } & 35.0 \text { degrees } \\
\text { Xray - window iriciderice angle } & 0.0 \text { degrees }
\end{array}
$$

\begin{tabular}{|c|c|c|c|c|c|}
\hline ELEMENT & \multicolumn{2}{|r|}{ WEIGHT } & PREC ISION & \multicolumn{2}{|r|}{ DXIDE } \\
\hline & K-RATIO** & PERCENT & ₹ SIGMA & FORMULA & FERCENT \\
\hline$A 1 \mathrm{KA}$ & 0. .1053 & 1. 68 & $0.1 E$ & Al 203 & 3.17 \\
\hline $\mathrm{Cl} \mathrm{KA}$ & 0.0173 & 3.30 & 0.13 & C1 & 3.30 \\
\hline$T_{i} K A$ & 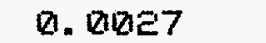 & 0.34 & $0 . \nabla 16$ & Tiae & Q. 56 \\
\hline Cr KA & Q. $8 B 70$ & 9.84 & 0. 18 & CrEDక & 14.38 \\
\hline Fe KA. & 0. & 0.94 & 0.09 & $F e O$ & 1. 30 \\
\hline $\mathrm{Ni} \mathrm{KA}$ & 0. 2583 & 27.61 & D. 34 & NiO & 35.14 \\
\hline Nb KA & D. 0964 & 10.90 & 1. $\nabla_{1}$ & Nbeos & 15.59 \\
\hline MO KA & Q. $15 \mathrm{EE}$ & 17.77 & 1.46 & $\operatorname{MaO} 3$ & EE. EE \\
\hline $0 \quad *$ & & 27.63 & & & \\
\hline TOTAL & & & & & 180.01 \\
\hline $\begin{array}{l}\text { DETERMINE } \\
\text { ITERATIO }\end{array}$ & $\begin{array}{ll}D \text { D BY } & \text { STOIO } \\
\text { ONS } & \text { EO }\end{array}$ & OMET & & & \\
\hline **NOTE: & $\begin{array}{l}K \text { KATIO }= \\
\text { where } R=\end{array}$ & $\begin{array}{l}\text { K-RATIO } x \\
\text { refererice }\end{array}$ & $\begin{array}{l}R \\
\text { (staridard)/r }\end{array}$ & $-x^{2}$ & les \\
\hline & NORMALIZ & ATION FAC & 1.890 & & \\
\hline
\end{tabular}

STANDARDLESS EDS ANALYSIS

( $Z$ AF CORRECTIONS VIA MAGIC $V$ ) 


$$
19-J \operatorname{ar}-1993 \quad 15: 47: \square 18
$$

Accelerating voltage

Bearn - sample iriciderice angle

Xray emergerice arigle

Xray - window inciderice argle

STANDARDLESS EDS ANALYSIS

(ZAF CORRECTIONS VIA MAGIC $V$ )

\section{ELEMENT \\ \& LINE}

A1 $K A$

CI KA

Ti $K A$

$\mathrm{Cr} K A$

Fe KA

$\mathrm{Ni} K A$

Zn KA

Nb KA

MO KA

Ce LA

Fb LA

D *

TOTAL
SCWD-17

30. $8 \mathrm{KeV}$

90.0 degrees

35. 0 degrees

Q. degrees
DXIDE

FERCENT

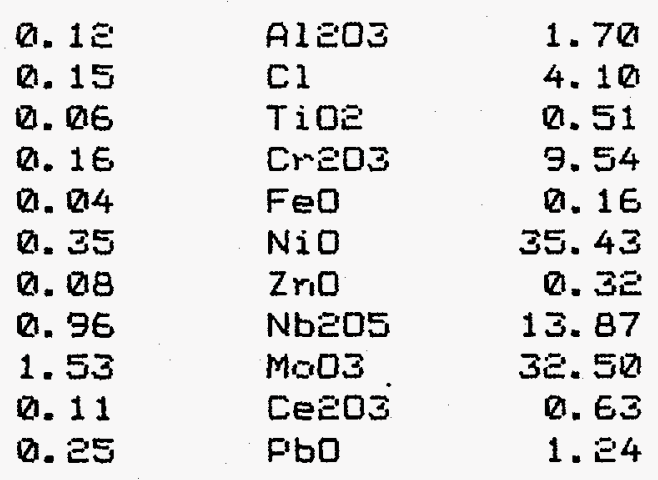

1018. Q1

* determined ey stoichiometry ITERATIONS EQ

**NOTE: K-RATIO $=K$ K-RATIO $\times R$

where $R=$ refererice (staridard)/refererice (sample)

NORMALIZATION FACTOR: $\quad 1.890$ 


$$
\begin{array}{lc}
\text { Acceleratirig voltage } & 30.0 \mathrm{KeV} \\
\text { Beam - sample incidence argle } & 90.0 \text { degrees } \\
\text { Xray emergerice arigle } & 35.0 \text { degrees } \\
\text { Xray - wiridow inciderice argle. } & \text { (0. degrees }
\end{array}
$$

\begin{tabular}{|c|c|c|c|c|c|c|}
\hline Al & KA & D. 82168 & 1.98 & ט. $\Xi 1$ & A1 203 & 3.74 \\
\hline $\mathrm{Cl}$ & $K A$ & ๑. & 0.41 & D1. 09 & $\mathrm{Cl}$ & D. 41 \\
\hline$T i$ & $K A$ & D. $\square \square B B$ & 1.11 & 0.11 & TiDe & 1.85 \\
\hline $\mathrm{Cr}^{2}$ & $K A$ & Q. 1367 & $15.7 E$ & 0.30 & Cr:Ẽ & 23. 13 \\
\hline $\mathrm{Fe}$ & $K A$. & Q. Q012 & Q. 14 & 0.05 & $F \in D$ & D. 18 \\
\hline $\mathrm{Ni}$ & KA & ‥ 1585 & $17.1 \Xi$ & 0.37 & NiO & 21.80 \\
\hline $\mathrm{Zn}$ & $K A$ & ฉ. $\square \square 33$ & B. 37 & 0.10 & Zno & Q. 46 \\
\hline $\mathrm{Nb}$ & $K A$ & ロ. EEE & 25.78 & 1.72 & NbE05 & 36.88 \\
\hline Mo & $K A$ & a. 18489 & 5.66 & $1 . \emptyset E$ & $\mathrm{MoO3}$ & 8.49 \\
\hline $\mathrm{Ce}$ & LA & Q. 10075 & Q. 87 & Q. 16 & Ceこ0З & $1.0 \Xi$ \\
\hline $\mathrm{Pb}$ & LA & Q. 0161 & E. 80 & D. $3 E$ & Pbo & E. 15 \\
\hline & * & & $\geq 8.8 \emptyset$ & & & \\
\hline
\end{tabular}

STANDARDLESS EDS ANALYSIS (ZAF CORRECTIONS VIA MAGIC $V$ )

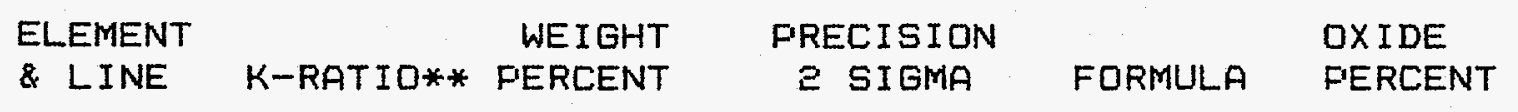

TOTAL

* determined by staichidMetry ITERATIONS . 20

**NOTE: K-RATIO $=K$ K-RATIO $\times R$

where $R=$ reference (staridard)/refererice (sample)

NORMALIZATION FACTOR: 1.891 


$$
\text { 19-Jar-1993 } 16: 09: 56 \quad \text { SCWD-19 }
$$

$$
\begin{array}{lc}
\text { Acceleratirig voltage } & 30.0 \mathrm{KeV} \\
\text { Beam - sample iriciderice arigle } & 90.0 \text { degrees } \\
\text { Xray emergence arigle } & 35.0 \text { degrees } \\
\text { Xray - window inciderice argle } & 0.0 \text { degrees }
\end{array}
$$

\begin{tabular}{|c|c|c|c|c|c|}
\hline $\begin{array}{l}\text { ELEMENT } \\
\text { \& LINE }\end{array}$ & K-RATIO** & $\begin{array}{l}\text { WEIGHT } \\
\text { FERCENT }\end{array}$ & $\begin{array}{l}\text { FRECISION } \\
=\text { SIGMA }\end{array}$ & FORMULA & $\begin{array}{l}\text { QXIDE } \\
\text { FERCENT }\end{array}$ \\
\hline $\mathrm{Cr} \mathrm{KA}$ & 0.0107 & 1.27 & D. 289 & Creo3 & 1.85 \\
\hline $\mathrm{Ni} K A$ & R. $338 n$ & E.5. 03 & 0.30 & $\mathrm{NiO}$ & 31.85 \\
\hline Mo KA & $0.3860^{\circ}$ & 44.19 & 1.90 & moos & $E E .30$ \\
\hline D * & & 29.51 & & & \\
\hline
\end{tabular}

STANDARDLESS EDS ANALYSIS

(ZAF CORRECTIONS VIA MAGIC V)

TOTAL

100. 00

* DeTERMINED EY STOICHIOMETRY
. ITERATIONS

**NOTE: K-RATIO $=K$-RATIO $\times R$ where $R=$ refererice (standard)/refererice (sample)

NORMALIZATION FACTOR: $\quad 1.464$ 


$$
\text { 19-Jar-1993 16:13:03 }
$$

Accelerating voltage

Beam - sample iriciderice arigle

Xray emergerice arigle

Xray - wiridow iriciderice arigle

STANDARDLESS EDS ANALYSIS

(ZAF CORRECTIONS VIA MAGIC $V$ )

\section{ELEMENT}

\&. LINE

AI $K A$

Ti KA

$\mathrm{Cr} K A$

$\mathrm{FE} K A$

$N i K A$

$\mathrm{Zr}, \mathrm{KA}$

Nb KA

MO KA

0 *

TOTAL
SCWD- $\approx \square$

$$
3 \square . ~ \mathrm{KeV}
$$

90. D degrees

35. 11 degrees

\begin{tabular}{|c|c|c|}
\hline & IT & FRECISION \\
\hline -RATIO* & FERCENT & E SIGMA \\
\hline
\end{tabular}

Q. degrees
Q. 01849
1. $\theta E$
Q. 28
ฉ.
2อ. 67
Q. 01019
1. 18
Q. 2597
จ. 0596
27. 85
7. 17
D. 0536
6. 23
0. 0468
5.53
27. 38

จ. 17

D. 0.125

D. $\Xi 4$

B. 09

0. 32

Q. $2 \mathrm{E}$

ט. 78

0. 92
A1 203
TIOE
Creaz
Feo
$\mathrm{NiO}$
Zrio
NbEOS
MaOJ
3. 44
0. 47
33. 13
1. 52
35.44
8. 79
8. 91
8. 30

100.01

* DETERMINED BY STOICHIOMETRY

ITERATIONS $\geq 0$

**NOTE: K-RATIO $=K$ K-RATIO $\times R$

where $R$ = refererice (staridard)/refererice (sample)

NORMALIZATION FACTOR: $\quad 1.894$ 


$$
\text { 19-Jar-1993 } 16: 16: 099 \text { SCWD-E1 }
$$

$$
\begin{array}{lc}
\text { Accelerating voltage } & 30.0 \mathrm{KeV} \\
\text { Bean - sample inciderice arigle } & 90.0 \text { degrees } \\
\text { Xray emergence angle } & 35.0 \text { degrees } \\
\text { Xray - wiridow iriciderice arigle } & 0.0 \text { degrees }
\end{array}
$$

STANDARDLESS EDS ANALYSIS

(ZAF CORRECTIONS VIA MAGIC $V$ )

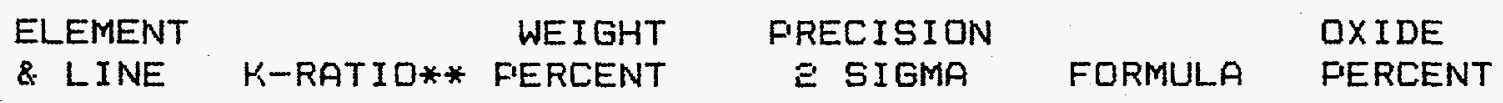

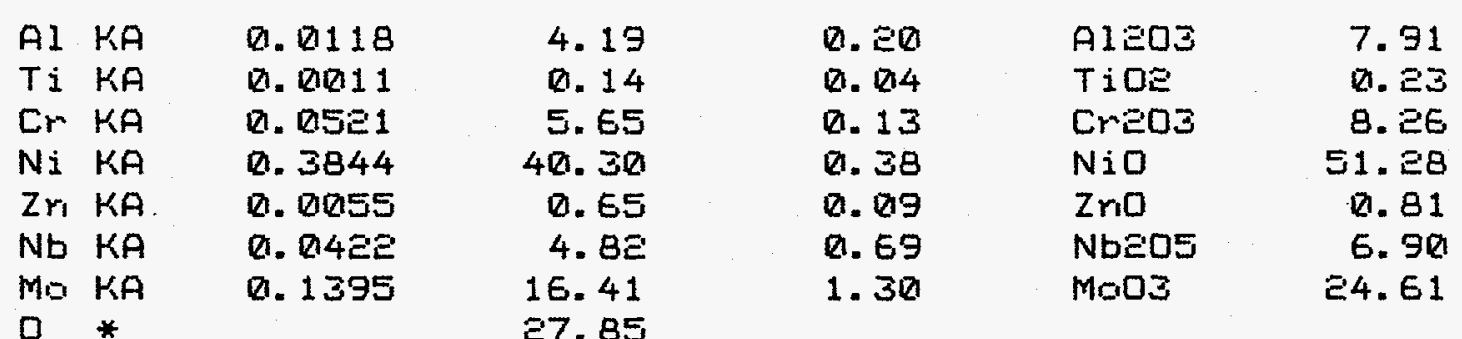

TOTAL

10a. 01

* DETERMINED EY STOICHIOMETRY

ITERATIONS 20

**NOTE: K-RATIO $=K$ K-RATIO $\times R$

where $R=$ reference (standard)/reference (sample)

NORMALIZATION FACTOR: 1.888 


$$
\text { 19-Jar-1993 1E: } 2010105
$$

Accelerating voltage

Beam - sample iriciderice arigle

Xray emergerice arigle

Xray - wiridaw iriciderice arigle

STANDARDLESS EDS ANALYSIS

(ZAF CORRECTIONS VIA MAGIC $V$ )

\section{ELEMENT \\ \& LINE}

Ti $K A$

Cr $K A$

$N$ N KA

ND KA

MO KA.

D *

TOTAL
SCWO-EE

30. $0 \mathrm{KeV}$

90. D degrees

35.0 degrees

Q. Q degrees
OXIDE

FRECISION

Z SIGMA

FORMULA

Q. 05

a. 38

Q. 018

Q. 44

D. $3 \Xi$

Q. 96

31.55
FERCENT

Tide
CrEa3
Nid
NuEdas
MaOJ

0. 93

94. 17

จ. 93

2. 53

1.44

* DETERMINED BY STOICHIOMETRY

ITERATIONS $\quad 20$

**NOTE: K-RATIO $=K-R A T I O ~ * R$

where $R=$ refererice (standard)/refererice (sample)

NORMALIZATION FACTOR: 1.669 


$$
\text { 19-Jar-1993 1E:EE:51 }
$$

Acceleratirg voltage

Beam - sample iriciderice argle

Xray emergence arigle

Xray - window iriciderice argle

\section{SCWO-EJ}

3Q. $Q \mathrm{KEV}$

3a. degrees

35. 17 degrees

Q. degrees

STANDARDLESS EDS ANALYSIS

(ZAF CORRECTIONS VIA MAGIC V)

\begin{tabular}{|c|c|c|c|c|c|}
\hline $\begin{array}{l}\text { ELEMENT } \\
\text { \&. LINE }\end{array}$ & a** & $\begin{array}{r}\text { WEIGHT } \\
\text { FERCENT }\end{array}$ & $\begin{array}{c}\text { FRECISION } \\
=\text { SIGMA }\end{array}$ & FDRMUU $A$ & QXIDE \\
\hline
\end{tabular}

\begin{tabular}{|c|c|c|c|}
\hline Al & $K A$ & Q. $\operatorname{Qn} 188$ & Е.5E \\
\hline CI & $K A$ & 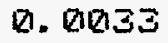 & Q. .49 \\
\hline $\mathrm{Ti}$ & $K A$ & D. $\square \square \square \in G$ & D. 6 \\
\hline $\mathrm{Cr}$ & $K A$ & Q. 4378 & $4 E .1$ \\
\hline $\mathrm{Fe}$ & $K A$ & 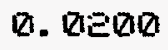 & 2. 4 \\
\hline $\mathrm{Ni}$ & $K A$ & a. 1094 & 1己. $E$ \\
\hline Nb & $K A$ & ด. ดีEE1 & E. 5 \\
\hline Mo & $K A$ & 0.0184 & E. 1 \\
\hline 0 & * & & 30.3 \\
\hline
\end{tabular}

TDTAL
B. 17
Q. DE
A1 203
Cl
4.84
D. DEE
D. 34
D. 11
a. 24
0. 58
Q. $G 2$
TiOe
D. 49
Creos
1. 10
FeO
$\mathrm{NiO}$
NbE05
67.40
3. 15
1E. 05
3.78
3. 29

1018.01

* DeTERMINED eY STOICHIOMETRY

ITERATIONS ED

**NOTE: K-RATIO $=K$-RATIO $\times R$

where $R=$ reference (staridard)/reference (sample)

NORMALIZATION FACTOR: 1.893 


$$
\text { 19-Jar-1993 } 16: 25: 18 \quad \text { SCWD-E4 }
$$

$$
\begin{array}{lc}
\text { Accelerating voltage } & 30.0 \text { KeV } \\
\text { Beam - sample imcidence argle } & 90.0 \text { degrees } \\
\text { Xray emergence angle } & 35.0 \text { degrees } \\
\text { Xray - window iricidence argle } & 0.0 \text { degrees }
\end{array}
$$

\begin{tabular}{|c|c|c|c|c|c|}
\hline $\begin{array}{l}\text { ELEMENT } \\
\& \text { LINE }\end{array}$ & K-RATIO** & $\begin{array}{l}\text { WEIGHT } \\
\text { FERCENT }\end{array}$ & $\begin{array}{l}\text { PRECISION } \\
=\text { SIGMA }\end{array}$ & FORMULA & $\begin{array}{l}\text { QXIDE } \\
\text { FERCENT }\end{array}$ \\
\hline $\mathrm{Al} \mathrm{KA}$ & Q. ฉロ९७7 & 3. $Q 10$ & Q. $1 E$ & A1:03 & 5.67 \\
\hline$T i K A$ & Q. 8097 & 1.01 & D. DE & TIOE & 1.68 \\
\hline$C r \quad K A$ & D. $41 \approx^{4}$ & 44.51 & 0.33 & CrED & $E \Xi . \not E \in$ \\
\hline Fe KA & Q. QIQZEE & $0.3=$ & Q. 05 & $\mathrm{FeD}$ & D. 41 \\
\hline$N i \quad K A$ & Q. 18834 & 9.56 & B. $E \square$ & $\mathrm{NiO}$ & $1 \Xi .1 E$ \\
\hline No KA & ด. $ด \geq 57$ & ‥ 98 & Q. 51 & Nbこ05 & 4. $\Xi E$ \\
\hline Ma KA & Q. $\square \in \square 4$ & 7.17 & D. 91 & MoO3 & 10.76 \\
\hline D * & & 31.45 & & & \\
\hline TOTAL & & & & & 100.00 \\
\hline $\begin{array}{l}\text { DETERMIN } \\
\text { ITERAT I }\end{array}$ & $\begin{array}{l}\text { ED BY STOICH } \\
\text { ONS } \\
\text { ONQ }\end{array}$ & BHIDMET & & & \\
\hline **NOTE: & $\begin{array}{l}K-\text { RATIO }=r \\
\text { where } R=r\end{array}$ & $\begin{array}{l}\text { K-RATIO } x \\
\text { refererice }\end{array}$ & $\begin{array}{l}\mathrm{R} \\
(\text { standard) } / r\end{array}$ & prerice & nple) \\
\hline
\end{tabular}

STANDARDLESS EDS ANALYSIS

(ZAF CORRECTIONS VIA MAGIC $V$ ) 


$$
\begin{array}{lc}
\text { Acceleratirg voltage } & 30.0 \mathrm{KeV} \\
\text { Heam - sample iriciderice argle } & 90.0 \text { degrees } \\
\text { Xray emergerice angle } & 35.0 \text { degrees } \\
\text { Xray - windaw iriciderice arigle } & 0.0 \text { degrees }
\end{array}
$$

\begin{tabular}{|c|c|c|c|c|c|}
\hline ELEMENT & \multirow[b]{2}{*}{ K-RATIO** } & WEIGHT & \multicolumn{2}{|l|}{ FRECISION } & \multirow{2}{*}{$\begin{array}{l}\text { OXIDE } \\
\text { FERCEN }\end{array}$} \\
\hline \& LINE & & PERCENT & E SIGMA & FORMULA & \\
\hline Al $\mathrm{KA}$ & Q. $\square \square=7$ & D. 89 & D. 11 & A1 203 & 1.69 \\
\hline Ti KA & 0. 9837 & Q1. 35 & Q1. 125 & TIOE & 0. 59 \\
\hline Cr $K A$ & 0.5105 & 53.30 & Q. 37 & CrED & 77.89 \\
\hline$F E \quad K A$ & Q. DEE 1 & Q. $\Xi E$ & D. D.E & $\mathrm{FeD}$ & Q. 34 \\
\hline$N i K A$ & 0.10143 & 12.10 & Q1. 24 & $\mathrm{NiO}$ & $15.4 Q$ \\
\hline Nb $K A$ & 0.0150 & 1.76 & 0. 43 & NBEOS & 2.51 \\
\hline Mo KA & Q. 8088 & 1.05 & 0.34 & $\operatorname{Mo03}$ & 1.58 \\
\hline 口 * & & 30.28 & & & \\
\hline
\end{tabular}

STANDARDLESS EDS ANALYSIS

(ZAF CORRECTIONS VIA MAGIC $V$ )

TOTAL

99.99

* Determined BY STOICHIDMETRY
ITERATIONS

**NOTE: K-RATIO $=K$-RATIO $\times R$

where $R=$ refererice (staridard)/reference (sample)

NORMALIZATION FACTOR: 1.893 